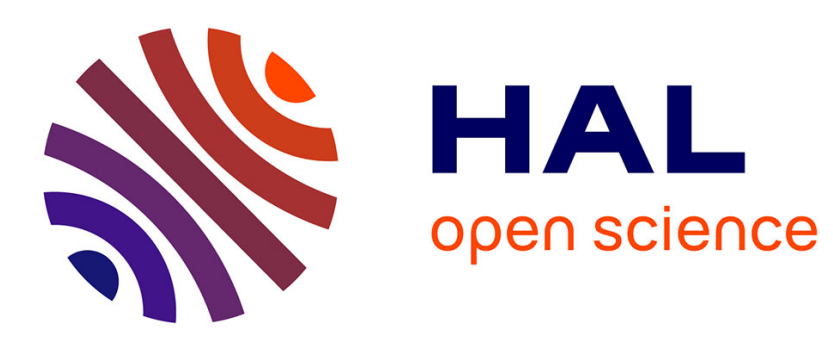

\title{
An all-regime Lagrange-Projection like scheme for the gas dynamics equations on unstructured meshes
}

Christophe Chalons, Mathieu Girardin, Samuel Kokh

\section{To cite this version:}

Christophe Chalons, Mathieu Girardin, Samuel Kokh. An all-regime Lagrange-Projection like scheme for the gas dynamics equations on unstructured meshes. 2014. hal-01007622v2

\section{HAL Id: hal-01007622 \\ https://hal.science/hal-01007622v2}

Preprint submitted on 17 Jun 2014

HAL is a multi-disciplinary open access archive for the deposit and dissemination of scientific research documents, whether they are published or not. The documents may come from teaching and research institutions in France or abroad, or from public or private research centers.
L'archive ouverte pluridisciplinaire HAL, est destinée au dépôt et à la diffusion de documents scientifiques de niveau recherche, publiés ou non, émanant des établissements d'enseignement et de recherche français ou étrangers, des laboratoires publics ou privés. 


\title{
An all-regime Lagrange-Projection like scheme for the gas dynamics equations on unstructured meshes
}

\author{
Christophe Chalons *
}

\author{
Mathieu Girardin ${ }^{\dagger \ddagger}$
}

Samuel Kokh ${ }^{\S}$

June 17,2014

\begin{abstract}
We propose an all regime Lagrange-Projection like numerical scheme for the gas dynamics equations. By all regime, we mean that the numerical scheme is able to compute accurate approximate solutions with an under-resolved discretization, i.e. a mesh size and time step much bigger than the Mach number $M$. The key idea is to decouple acoustic and transport phenomenon and then alter the numerical flux in the acoustic approximation to obtain a uniform truncation error in term of $M$. This modified scheme is conservative and endowed with good stability properties with respect to the positivity of the density and the internal energy. A discrete entropy inequality under a condition on the modification is obtained thanks to a reinterpretation of the modified scheme in the Harten Lax and van Leer formalism. A natural extension to multi-dimensional problems discretized over unstructured mesh is proposed. Then a simple and efficient semi implicit scheme is also proposed. The resulting scheme is stable under a CFL condition driven by the (slow) material waves and not by the (fast) acoustic waves and so verifies the all regime property. Numerical evidences are proposed and show the ability of the scheme to deal with tests where the flow regime may vary from low to high Mach values.
\end{abstract}

\section{Introduction}

In this paper, we consider the system of gas dynamics in two space dimension in situations when the flow regime may vary in terms of Mach number $M$ across the computational domain. We propose a collocated Finite Volume method that addresses two important issues.

The first issue concerns the lack of accuracy in the low Mach regime of Godunov-type schemes. While these methods performs well at capturing shocks, they may generate spurious numerical diffusion when they are used for simulating low Mach flows over relatively coarse mesh, i.e. mesh size much bigger the Mach number. Improvements of Godunov-type schemes more generally of collocated methods have been proposed by many authors like $[29,16,21,5,9,17,28,18,24,23,14,11,8,19$. The analysis of these authors may rely on different arguments like the analysis of the viscosity matrix [29], an asymptotic expansion in terms of Mach number [16], a detailed study in [11] that seek for invariance properties of the numerical scheme transposing the framework of Schochet [25] to the discrete setting, and also an analysis based on the so-called Asymptotic Preserving property [20] in [19]. Nevertheless the resulting cure usually boils down to reduce the numerical diffusion in the momentum equation for low Mach number values.

The second problem we address deals with subsonic flow when the fluid velocity is slow and the acoustic waves are not driving phenomenons. In this case, the Courant-Friedrichs-Lewy (CFL) condition on the time step for explicit Godunov-type methods that involves the (fast) acoustic wave velocity may lead to very small time steps choices and thus costly computations. It seems natural to seek for numerical schemes that enable the use of a large time steps that are not constrained by the sound velocity. This question has been examined

\footnotetext{
* christophe.chalons@uvsq.fr LMV - UMR 8100, Univ. Versailles Saint-Quentin-en-Yvelines, UFR des Sciences, Bâtiment Fermat, 45 avenue des Etats-Unis, 78035 Versailles cedex, France.

tmathieu.girardin@cea.fr DEN/DANS/DM2S/STMF/LMEC CEA Saclay, bât. 454 PC 47, 91191 Gif sur Yvette Cedex, France.

${ }^{\ddagger}$ LRC MANON, Laboratoire de Recherche Conventionné CEA/DEN/DANS/DM2S and UPMC-CNRS/LJLL.

$\S$ samuel.kokh@cea.fr. Maison de la Simulation USR 3441, Digiteo Labs, bât. 565, PC 190, CEA Saclay, 91191 Gif-sur-Yvette, France.

ๆDEN/DANS/DM2S/STMF, CEA Saclay, 91191 Gif-sur-Yvette, France.
} 
by several authors like [21, 7, 8, 9, 19] (see also [4, 6]) who derived mixed implicit-explicit strategies that allows to choose the time step independently of the Mach Number.

Numerical schemes that can tackle both issues, namely: accuracy for mesh sizes that do not depend on the Mach number and also stability for time steps that are not constrained by the Mach value are usually referred to as all regime, like the methods proposed by [21, 7, 8, 9, 19.

In the present work, we first propose an operator splitting strategy that allows to decouple the acoustic and the transport phenomenons. The approximation algorithm is split into two steps: an acoustic step and a transport step. For one-dimensional problems, this strategy is equivalent to an explicit Lagrange-Projection [15, 13. method, however the present splitting does not involve any moving Lagrangian mesh and can be naturally expressed for multi-dimensional problems. Following simple lines inspired by [11, 10] we investigate the dependence of the truncation error with respect to the Mach number. Let us mention that our study does not involve a Taylor expansion in the vicinity of the zero-Mach limit, nor a near-divergence free condition for the velocity field. Although this analysis is by no mean a thorough explanation of the low Mach regime behavior of our solver, it is enough to suggest simple means to obtain a truncation error with a uniform dependence on the Mach number for $M<1$. The cure simply relies on modifying the pressure terms in the flux of the acoustic operator that is coherent with the correction proposed by [11, 10, 18, 24, 14. Although this modified scheme is based on a modified flux definition, one can shows that it can also be rephrased as a simple approximate Riemann solver in the sense of Harten, Lax and van Leer [17 that is consistent with the integral form of the gas dynamics equation. This scheme is endowed with good stability properties under a CFL condition that involves the Mach number as the time step is still constrained by the sound velocity.

We propose to circumvent this time-step restriction by implementing a mixed implicit-explicit method following the ideas developped by [6] for one-dimensional problems using a genuine Lagrange-Projection framework. This idea was also used in [4] and consists in using an implicit update for the acoustic step and an explicit march in time for the transport step. This enables stability under a CFL condition that only involves the (slow) material waves without the (fast) acoustic waves. Finally, let us mention that the overall procedure is a conservative discretization that relies on a Suliciu relaxation approach 27] that allows to cope with compressible fluids equipped with very general Equation of State (EOS).

The paper is structured as follows: we first present the operator splitting considering only one-dimensional problems. Then we study the behavior of the scheme in the low Mach regime. This allows to lead to an explicit corrected scheme for the sole acoustic step that preserves the accuracy of the scheme at low Mach. Interestingly, we show that this flux-based corrected method may be expressed thanks to an approximate Riemann solver for the acoustic step. Next and thanks to this property, we investigate the ability of the corrected scheme to satisfy to a discrete entropy inequality. Afterwards, we present the extension of the operator splitting method to unstructured meshes either with a semi-implicit or full-explicit march in time. Finally we present numerical results involving low Mach and multi-regime flows.

\section{Governing equations}

We are interested in the two-dimensional gas dynamics equations

$$
\left\{\begin{array}{r}
\partial_{t} \rho+\nabla \cdot(\rho \mathbf{u})=0 \\
\partial_{t}(\rho \mathbf{u})+\nabla \cdot(\rho \mathbf{u} \otimes \mathbf{u})+\nabla p=0 \\
\partial_{t}(\rho E)+\nabla \cdot[(\rho E+p) \mathbf{u}]=0
\end{array}\right.
$$

where $\rho, \mathbf{u}=\left(u_{1}, u_{2}\right)^{t}, E$ denote respectively the density, the velocity vector and the total energy of the fluid. Let $e=E-\frac{|\mathbf{u}|^{2}}{2}$ be the specific internal energy of the fluid and $s$ its specific entropy. We note $\tau=1 / \rho$ and we suppose given an Equation of State (EOS) through the mapping $(\tau, s) \mapsto e^{\mathrm{EOS}}$ which satisfies to the usual Weyl assumptions 30.

$$
\begin{array}{rrrrr}
\partial_{\tau} e^{\mathrm{EOS}} & <0, & \partial_{s} e^{\mathrm{EOS}}>0, & \partial_{\tau \tau} e^{\mathrm{EOS}}>0 \\
\partial_{s s} e^{\mathrm{EOS}}>0, & \partial_{\tau \tau} e^{\mathrm{EOS}} \partial_{s s} e^{\mathrm{EOS}}>\left(\partial_{\tau s} e^{\mathrm{EOS}}\right)^{2}, & \partial_{\tau \tau \tau} e^{\mathrm{EOS}}<0 .
\end{array}
$$

The entropy $s=s^{\operatorname{EOS}}(\tau, e)$ verifies $e=e^{\operatorname{EOS}}(\tau, s)$ thanks to (2) and we can define the pressure $p=-\partial_{\tau} e^{\mathrm{EOS}}$ and the sound velocity $c=\tau \sqrt{\partial_{\tau \tau} e^{\mathrm{EOS}}}$. The above assumptions imply that $(\tau, s) \mapsto e^{\mathrm{EOS}}$ and $(\tau, e) \mapsto-s^{\mathrm{EOS}}$ 
are strictly convex. Using a slight abuse of notation, we shall also consider $p$ as a function of $(\tau, e)$ and note $p=p^{\mathrm{EOS}}(\tau, e)$.

\section{Acoustic/transport operator splitting strategy for the one-dimensional problem}

In this section we will consider for the sake of simplicity one-dimensional problems and propose a two-step approximation strategy based on an operator splitting. The aim of this splitting is to decouple acoustic and transport phenomena. Using this guideline we will propose an explicit numerical solver. We shall propose two simple extensions of this method to two-dimensional problems discretized over unstructured grids using either an explicit or a semi-implicit time update in section 5.4 .

Before going any further, we introduce classical notations for the one-dimensional setting: let $\Delta t>0$ and $\Delta x>0$ be respectively the time and space steps. We define the Eulerian mesh interfaces $x_{j+1 / 2}=j \Delta x$ for $j \in \mathbb{Z}$, and the intermediate times $t^{n}=n \Delta t$ for $n \in \mathbb{N}$. If $b$ is a fluid parameter, in the sequel, we will note $b_{j}^{n}\left(\right.$ resp. $\left.b_{j}^{n+1}\right)$ the approximate value $b$ respectively within the $j^{\text {th }}$ cell $\left[x_{j-1 / 2}, x_{j+1 / 2}\right)$ at instant $t=t^{n}($ resp. $\left.t=t^{n+1}\right)$.

For one-dimensional problems, (1) supplemented with a passive scalar variable $v$ (that will account for the transverse velocity in two-dimensional problems) reads

$$
\left\{\begin{aligned}
\partial_{t} \rho+\partial_{x}(\rho u) & =0 \\
\partial_{t}(\rho u)+\partial_{x}\left(\rho u^{2}+p\right) & =0 \\
\partial_{t}(\rho v)+\partial_{x}(\rho u v) & =0 \\
\partial_{t}(\rho E)+\partial_{x}[(\rho E+p) u] & =0
\end{aligned}\right.
$$

Our discretization strategy of (3) consists in approximating successively the solutions of the following systems (4) and (5) where

$$
\left\{\begin{aligned}
\partial_{t} \rho+\rho \partial_{x} u & =0 \\
\partial_{t}(\rho u)+\rho u \partial_{x} u+\partial_{x} p & =0 \\
\partial_{t}(\rho v)+\rho v \partial_{x} u & =0 \\
\partial_{t}(\rho E)+\rho E \partial_{x} u+\partial_{x}(p u) & =0
\end{aligned}\right.
$$

and

$$
\left\{\begin{aligned}
\partial_{t} \rho+u \partial_{x} \rho & =0 \\
\partial_{t}(\rho u)+u \partial_{x}(\rho u) & =0 \\
\partial_{t}(\rho v)+u \partial_{x}(\rho v) & =0 \\
\partial_{t}(\rho E)+u \partial_{x}(\rho E) & =0 .
\end{aligned}\right.
$$

In the sequel, system (4) and (5) will be respectively referred to as the acoustic system and the transport system.

Given a fluid state $(\rho, \rho u, \rho v, \rho E)_{j}^{n}, j \in \mathbb{Z}$ at instant $t^{n}$, this splitting algorithm can be decomposed as follows.

1. Update the fluid state $(\rho, \rho u, \rho v, \rho E)_{j}^{n}$ to the value $(\rho, \rho u, \rho v, \rho E)_{j}^{n+1-}$ by approximating the solution of (4);

2. Update the fluid state $(\rho, \rho u, \rho v, \rho E)_{j}^{n+1-}$ to the value $(\rho, \rho u, \rho v, \rho E)_{j}^{n+1}$ by approximating the solution of 5 .

\subsection{Properties and approximation of the one-dimensional acoustic system}

First, we notice that the acoustic system (4) reads equivalently

$$
\partial_{t} \tau-\tau \partial_{x} u=0, \quad \partial_{t} u+\tau \partial_{x} p=0, \quad \partial_{t} v=0, \quad \partial_{t} E+\tau \partial_{x}(p u)=0 .
$$


The acoustic system (6) is a quasilinear system that can be simply checked to be strictly hyperbolic. Indeed, the Jacobian of the system (6) has three eigenvalues $\left(\lambda_{1}, \lambda_{2}, \lambda_{3}\right)=(-c, 0,+c)$. The waves associated with $\lambda_{1}$ and $\lambda_{3}$ are genuinely nonlinear waves while the wave of velocity $\lambda_{2}=0$ is a stationnary contact discontinuity.

In order to derive an update process from $(\rho, \rho u, \rho v, \rho E)_{j}^{n}$ to $(\rho, \rho u, \rho v, \rho E)_{j}^{n+1-}$, we will perform several approximations. We notice that for a smooth solution (6) we also have $\partial_{t} p+\tau(\rho c)^{2} \partial_{x} u=0$ and we thus choose to perform a Suliciu-type approximation of (6) for $t \in\left[t^{n}, t^{n}+\Delta t\right)$ by introducing a surrogate pressure $\Pi$ and considering the relaxed system

$$
\left\{\begin{aligned}
\partial_{t} \tau-\tau \partial_{x} u & =0, \\
\partial_{t} u+\tau \partial_{x} \Pi & =0, \\
\partial_{t} v & =0, \\
\partial_{t} E+\tau \partial_{x}(\Pi u) & =0, \\
\partial_{t} \Pi+\tau a^{2} \partial_{x} u & =\nu(\Pi-p),
\end{aligned}\right.
$$

where $a>0$ is parameter whose choice will be specified later. In the regime $\nu \rightarrow+\infty$ we formally recover (6). In our numerical solver context, we classically mimic the $\nu \rightarrow+\infty$ regime enforcing at each time step $\Pi_{j}^{n}=p^{\mathrm{EOS}}\left(\tau_{j}^{n}, e_{j}^{n}\right)$ and then solving (7) with $\nu=0$.

At last, for $t \in\left[t^{n}, t^{n}+\Delta t\right)$ we choose to approximate $\tau(x, t) \partial_{x}$ by $\tau\left(x, t^{n}\right) \partial_{x}$ in (7). If one introduces the mass variable $m$ defined by $\mathrm{d} m=\rho\left(x, t^{n}\right) \mathrm{d} x$ our approximation of (6) (up to an abuse of notation) can be expressed in the following fully conservative form

$$
\partial_{t} \mathbf{W}+\partial_{m} \mathbf{F}(\mathbf{W})=0
$$

where $\mathbf{W}=(\tau, u, v, E, \Pi)^{T}$ and $\mathbf{F}(\mathbf{W})=\left(-u, \Pi, 0, \Pi u, a^{2} u\right)^{T}$. Let us remark that $(8)$ is consistent with a Suliciu relaxation of the gas dynamics equation written in Lagrangian coordinates using a mass variable formulation. The solution of the Riemann problem associated with (8) can be derived explicitly (see section C). This allows to write an exact Godunov solver for (8) that turns out to be an approximate Riemann solver for (6) following the Harten-Lax-van Leer formalism (see section B and 17, 1]). It provides us with the update formula

$$
\left\{\begin{aligned}
\mathbf{W}_{j}^{n+1-} & =\mathbf{W}_{j}^{n}-\frac{\Delta t}{\Delta x}\left(\mathbf{F}_{j+1 / 2}-\mathbf{F}_{j-1 / 2}\right), \\
\mathbf{F}_{j+1 / 2} & =\mathbf{F}\left(\mathbf{W}_{j}^{n}, \mathbf{W}_{j+1}^{n}\right), \\
\mathbf{F}\left(\mathbf{W}_{L}, \mathbf{W}_{R}\right) & =\left(-u^{*}, \Pi^{*}, 0, \Pi^{*} u^{*}, a^{2} u^{*}\right)^{T},
\end{aligned}\right.
$$

where

$$
\left\{\begin{array}{l}
u^{*}=\frac{\left(u_{R}+u_{L}\right)}{2}-\frac{1}{2 a}\left(\Pi_{R}-\Pi_{L}\right), \\
\Pi^{*}=\frac{\left(\Pi_{R}+\Pi_{L}\right)}{2}-\frac{a}{2}\left(u_{R}-u_{L}\right) .
\end{array}\right.
$$

The update of the conservative variables is obtained by setting $\rho_{j}^{n+1-}=1 / \tau_{j}^{n+1-},(\rho u)_{j}^{n+1-}=\rho_{j}^{n+1-} \times u_{j}^{n+1-}$, $(\rho v)_{j}^{n+1-}=\rho_{j}^{n+1-} \times v_{j}^{n+1-}$ and $(\rho E)_{j}^{n+1-}=\rho_{j}^{n+1-} \times E_{j}^{n+1-}$. This can be summed up by the following update formulas

$$
\left\{\begin{aligned}
L_{j} \rho_{j}^{n+1-} & =\rho_{j}^{n}, \\
L_{j}(\rho u)_{j}^{n+1-} & =(\rho u)_{j}^{n}-\frac{\Delta t}{\Delta x}\left(\Pi_{j+1 / 2}^{*}-\Pi_{j-1 / 2}^{*}\right), \\
L_{j}(\rho v)_{j}^{n+1-} & =(\rho v)_{j}^{n}, \\
L_{j}(\rho E)_{j}^{n+1-} & =(\rho E)_{j}^{n}-\frac{\Delta t}{\Delta x}\left(\Pi_{j+1 / 2}^{*} u_{j+1 / 2}^{*}-\Pi_{j-1 / 2}^{*} u_{j-1 / 2}^{*}\right), \\
L_{j} & =1+\frac{\Delta t}{\Delta x}\left(u_{j+1 / 2}^{*}-u_{j-1 / 2}^{*}\right) .
\end{aligned}\right.
$$

Let us remark that (9) also proposes an update relation for $\Pi$. However in this case $\Pi$ is just a disposable intermediate value whose role only consists in providing a formula for the interface pressure terms and the udpated value $\Pi_{k}^{n+1-}$ will be discarded. Indeed in this explicit scheme, $\Pi$ is updated after each time step by 
the equilibrium formula $\Pi_{j}^{n}=p^{\mathrm{EOS}}\left(\tau_{j}^{n}, e_{j}^{n}\right)$. However, this will no longer be the case for semi-implicit strategy as we will see in section 5.4 .

Let us finally note that the relaxation scheme (9) is equivalent to the acoustic scheme [12]. In order to avoid numerical instabilities, the parameter $a$ must complies with the subcharacteristic condition

$$
a>\max \rho c,
$$

for all possible values of $\rho c$ when considering a solution of the equilibrium system (6). In practice we will choose a value $a_{L R}$ for each interface by setting

$$
a_{L R}=K \max \left(\rho_{L}^{n} c_{L}^{n}, \rho_{R}^{n} c_{R}^{n}\right),
$$

where $K \geq 1, L R=j+1 / 2, L=j$ and $R=j+1$. We refer the reader to [1, 3, 2, 13] and the reference therein for more details.

\subsection{Properties and approximation of the one-dimensional transport system}

The transport system equation discretization is quite simple. Indeed, system (5) is a quasi-linear hyperbolic system that only involves the transport of the conservative variables with the velocity $u$. We choose to approximate the solution of (5) thanks to a standard upwind Finite-Volume approximation for $\varphi \in\{\rho, \rho u, \rho v, \rho E\}$

$$
\varphi_{j}^{n+1}=\varphi_{j}^{n+1-}-\frac{\Delta t}{\Delta x}\left(u_{j+1 / 2}^{*} \varphi_{j+1 / 2}^{n+1-}-u_{j-1 / 2}^{*} \varphi_{j-1 / 2}^{n+1-}\right)+\frac{\Delta t}{\Delta x} \varphi_{j}^{n+1-}\left(u_{j+1 / 2}^{*}-u_{j-1 / 2}^{*}\right),
$$

where

$$
\varphi_{j+1 / 2}^{n+1-}=\left\{\begin{array}{l}
\varphi_{j}^{n+1-}, \text { if } u_{j+1 / 2}^{*} \geq 0 \\
\varphi_{j+1}^{n+1-}, \text { if } u_{j+1 / 2}^{*}<0 .
\end{array}\right.
$$

Let us finally remark that 14 can be recast into

$$
\varphi_{j}^{n+1}=\varphi_{j}^{n+1-} L_{j}+\frac{\Delta t}{\Delta x}\left(u_{j+1 / 2}^{*} \varphi_{j+1 / 2}^{n+1-}-u_{j-1 / 2}^{*} \varphi_{j-1 / 2}^{n+1-}\right) .
$$

\subsection{Properties of the operator splitting scheme}

We present here a few properties of the operator splitting scheme defined by (9) and (14). Let us first remark that this algorithm performs the same update as a classical Lagrange-Remap (or equivalently Lagrange-Projection) algorithm for one-dimensional problems (see appendix A) although the design of our algorithm does not involve a moving mesh for following the variables in a Lagrangian reference frame. This feature will be the key element of the multi-dimensional extension of the present scheme. It is also interesting to mention that the operator splitting strategy also provided a mean of treating the waves of the gas dynamics system (3) separately: the acoustic step only involves acoustic waves while freezing the transport waves. The transport step only deals with the contact discontinuity of the material transport. Let us mention that a similar operator splitting was used in [?].

The overall update from variable at instant $t^{n}$ to variables at instant $t^{n+1}$ is fully conservative with respect to $\rho, \rho u, \rho v$ and $\rho E$. Indeed, we have

$$
\left\{\begin{aligned}
\rho_{j}^{n+1} & =\rho_{j}^{n}+\frac{\Delta t}{\Delta x}\left(u_{j+1 / 2}^{*} \rho_{j+1 / 2}^{n+1-}-u_{j-1 / 2}^{*} \rho_{j-1 / 2}^{n+1-}\right), \\
(\rho u)_{j}^{n+1} & =(\rho u)_{j}^{n}+\frac{\Delta t}{\Delta x}\left(u_{j+1 / 2}^{*}(\rho u)_{j+1 / 2}^{n+1-}+\Pi_{j+1 / 2}^{*}-u_{j-1 / 2}^{*}(\rho u)_{j-1 / 2}^{n+1-}-\Pi_{j-1 / 2}^{*}\right), \\
(\rho v)_{j}^{n+1} & =(\rho v)_{j}^{n}+\frac{\Delta t}{\Delta x}\left(u_{j+1 / 2}^{*}(\rho v)_{j+1 / 2}^{n+1-}-u_{j-1 / 2}^{*}(\rho v)_{j-1 / 2}^{n+1-}-\right), \\
(\rho E)_{j}^{n+1} & =(\rho E)_{j}^{n}+\frac{\Delta t}{\Delta x}\left(u_{j+1 / 2}^{*}(\rho E)_{j+1 / 2}^{n+1-}+\Pi_{j+1 / 2}^{*} u_{j+1 / 2}^{*}-u_{j-1 / 2}^{*}(\rho E)_{j-1 / 2}^{n+1-}-\Pi_{j-1 / 2}^{*} u_{j-1 / 2}^{*}\right) .
\end{aligned}\right.
$$

The scheme (9)- 10 for the acoustic step is stable under the Courant-Friedrichs-Lewy (CFL) condition

$$
\frac{\Delta t}{\Delta x} \max _{j \in \mathbb{Z}}\left(\max \left(\tau_{j}^{n}, \tau_{j+1}^{n}\right) a_{j+1 / 2}\right) \leq \frac{1}{2} .
$$


If one notes $b^{ \pm}=\frac{b \pm|b|}{2}$, then a classical result states that the CFL condition associated with the transport scheme (14) reads

$$
\Delta t \max _{j \in \mathbb{Z}}\left(\left(u_{j-\frac{1}{2}}^{*}\right)^{+}-\left(u_{j+\frac{1}{2}}^{*}\right)^{-}\right)<\Delta x .
$$

Entropy-related stability properties of the scheme will be examined in section 5.3 .

One can also remark that both the acoustic steps and the transport steps are achieved thanks to genuine Godunov solvers applied to simplified subsystems.

\section{Behavior of the scheme with respect to the Mach regime}

We are now interested in the behavior of the numerical scheme with respect to the variations of the Mach regime. In order to characterize the Mach regime of the flow, we consider a classical rescaling of the equations (3): let us introduce the following non-dimensional quantities:

$$
\tilde{x}=\frac{x}{L}, \quad \tilde{t}=\frac{t}{T}, \quad \tilde{\rho}=\frac{\rho}{\rho_{0}}, \quad \tilde{u}=\frac{u}{u_{0}}, \quad \tilde{v}=\frac{v}{v_{0}}, \quad \tilde{e}=\frac{e}{e_{0}}, \quad \tilde{p}=\frac{p}{p_{0}}, \quad \tilde{c}=\frac{c}{c_{0}} .
$$

The parameters $L, T, u_{0}=v_{0}=\frac{L}{T}, \rho_{0}, e_{0}=p_{0} \rho_{0}, p_{0}$ and $c_{0}=\sqrt{\frac{p_{0}}{\rho_{0}}}$ denote respectively a characteristic length, time, velocity, density, internal energy, pressure and sound speed. If $M=\frac{u_{0}}{c_{0}}$ is the so-called Mach-number then system (3) reads

$$
\left\{\begin{aligned}
\partial_{\tilde{t}} \tilde{\rho}+\partial_{\tilde{x}}(\tilde{\rho} \tilde{u}) & =0, \\
\partial_{\tilde{t}}(\tilde{\rho} \tilde{u})+\partial_{\tilde{x}}\left(\tilde{\rho} \tilde{u}^{2}\right)+\frac{1}{M^{2}} \partial_{\tilde{x}} \tilde{p} & =0, \\
\partial_{\tilde{t}}(\tilde{\rho} \tilde{v})+\partial_{\tilde{x}}(\tilde{\rho} \tilde{u} \tilde{v}) & =0, \\
\partial_{\tilde{t}}(\tilde{\rho} \tilde{E})+\partial_{\tilde{x}}[(\tilde{\rho} \tilde{E}+\tilde{p}) \tilde{u}] & =0,
\end{aligned}\right.
$$

where $\tilde{E}=\tilde{e}+\frac{1}{2} M^{2} \tilde{u}^{2}$. For a given small value of the Mach number, we distinguish two cases:

- the term $\partial_{\tilde{x}} \tilde{p}$ remains of magnitude $O\left(M^{2}\right)$. Then the variations of $\tilde{\rho} \tilde{u}$ are of order 1 which implies that all the tilde variables will remain of order 1 . We shall refer this case as the low Mach regime;

- the term $\partial_{\tilde{x}} \tilde{p}$ does not remain of magnitude $O\left(M^{2}\right)$. Then the variations of $\tilde{\rho} \tilde{u}$ will reach a magnitude $O(1 / M)$ or $O\left(1 / M^{2}\right)$. These large magnitude variations of the momentum will induce a growth of the Mach number and thus a change of Mach regime.

Before going any further, let us underline that in the present approach we do not intend to study the behavior of the rescaled system (3) in the limit regime $M \rightarrow 0$. This delicate question has been widely investigated over the past years and is still a rich field of research [16, 11, 19. We focus here on a simpler task that consists in examining the consistency of a rescaled approximate solution provided by the splitting operator algorithm with the solution of 20 in the low Mach regime. The framework we will place ourselves in does not require sophisticated hypotheses and may deal with the evaluation of a local behavior of the solution (a few neighbouring cells in the discrete setting). More precisely, if one considers smooth solutions of (20) and considers the truncation error of the rescaled numerical scheme in the sense of Finite Difference, how does it depends on $\mathrm{M}$ in the low Mach regime?

Introducing the rescaling defined earlier into 10 we get

$$
\tilde{u}_{j+1 / 2}^{*}=\frac{1}{2}\left(\tilde{u}_{j}^{n}+\tilde{u}_{j+1}^{n}\right)-\frac{1}{2 \tilde{a}_{j+1 / 2} M}\left(\tilde{\Pi}_{j+1}^{n}-\tilde{\Pi}_{j}^{n}\right), \quad \tilde{\Pi}_{j+1 / 2}^{*}=\frac{1}{2}\left(\tilde{\Pi}_{j}^{n}+\tilde{\Pi}_{j+1}^{n}\right)-\frac{\tilde{a}_{j+1 / 2} M}{2}\left(\tilde{u}_{j+1}^{n}-\tilde{u}_{j}^{n}\right),
$$


for 11 we have

$$
\left\{\begin{aligned}
\tilde{L}_{j} \tilde{\rho}_{j}^{n+1-} & =\tilde{\rho}_{j}^{n} \\
\tilde{L}_{j}(\tilde{\rho} \tilde{u})_{j}^{n+1-} & =(\tilde{\rho} \tilde{u})_{j}^{n}-\frac{\Delta \tilde{t}}{M^{2} \Delta \tilde{x}}\left(\tilde{\Pi}_{j+1 / 2}^{*}-\tilde{\Pi}_{j-1 / 2}^{*}\right), \\
\tilde{L}_{j}(\tilde{\rho} \tilde{v})_{j}^{n+1-} & =(\tilde{\rho} \tilde{v})_{j}^{n}, \\
\tilde{L}_{j}(\tilde{\rho} \tilde{E})_{j}^{n+1-} & =(\tilde{\rho} \tilde{E})_{j}^{n}-\frac{\Delta \tilde{t}}{\Delta \tilde{x}}\left(\tilde{\Pi}_{j+1 / 2}^{*} \tilde{u}_{j+1 / 2}^{*}-\tilde{\Pi}_{j-1 / 2}^{*} \tilde{u}_{j-1 / 2}^{*}\right), \\
\tilde{L}_{j} & =L_{j}=1+\frac{\Delta \tilde{t}}{\Delta \tilde{x}}\left(\tilde{u}_{j+1 / 2}^{*}-\tilde{u}_{j-1 / 2}^{*}\right),
\end{aligned}\right.
$$

and finally if $\tilde{\varphi} \in\{\tilde{\rho}, \tilde{\rho} \tilde{u}, \tilde{\rho} \tilde{v}, \tilde{\rho} \tilde{E}\}$ the rescaling of 15 , reads

$$
\frac{1}{\Delta \tilde{t}}\left(\tilde{\varphi}_{j}^{n+1}-\tilde{L}_{j} \tilde{\varphi}_{j}^{n+1-}\right)+\frac{1}{\Delta \tilde{x}}\left(\tilde{\varphi}_{j+1 / 2}^{n+1-} \tilde{u}_{j+1 / 2}^{*}-\tilde{u}_{j-1 / 2}^{*} \tilde{\varphi}_{j-1 / 2}^{n+1-}\right)=0 .
$$

Note that the CFL restriction of the acoustic step reads now

$$
\frac{\Delta \tilde{t}}{\Delta \tilde{x}} \max \left(\tilde{\tau}_{j}^{n}, \tilde{\tau}_{j+1}^{n}\right) \tilde{a}_{j+1 / 2}^{n} \leq \frac{M}{2},
$$

while the CFL restriction associated with the transport step is

$$
\left(\left(\tilde{u}_{j-1 / 2}^{*}\right)^{+}-\left(\tilde{u}_{j+1 / 2}^{*}\right)^{-}\right) \frac{\Delta \tilde{t}}{\Delta \tilde{x}} \leq 1 .
$$

In order to evaluate the truncation error (in the Finite Difference sense) in the low Mach regime, we use the classical tool of equivalent equations. Let $(\tilde{x}, \tilde{t}) \mapsto \tilde{b}$ be a parameter of (rescaled) functions that describe a smooth flow. With a classical slight abuse of notation, we consider that $\tilde{\varphi}\left(x_{j}, t^{n}\right)=\tilde{\varphi}_{j}^{n}$ so that we can substitute these functions into the discrete update formula when $\tilde{\varphi} \in\{\tilde{\rho}, \tilde{u}, \tilde{v}, \tilde{E}, \tilde{\Pi}\}$. We suppose that we are in low Mach regime, namely $\partial_{\tilde{x}} \tilde{p}=O\left(M^{2}\right)$. This hypothesis yields that $\tilde{\Pi}_{j+1}^{n}=\tilde{\Pi}_{j}^{n}+O\left(M^{2} \Delta \tilde{x}\right)$ for the discrete unknowns. We have the following result.

Proposition 1. In the low Mach regime, the rescaled discretization of the acoustic step is consistent with

$$
\begin{aligned}
\partial_{\tilde{t}} \tilde{\tau}-\tilde{\tau} \partial_{\tilde{x}} \tilde{u} & =O(\Delta \tilde{t})+O(M \Delta \tilde{x}), & \partial_{\tilde{t}} \tilde{u}+\frac{\tilde{\tau}}{M^{2}} \partial_{\tilde{x}} \tilde{p} & =O(\Delta \tilde{t})+O\left(\frac{\Delta \tilde{x}}{M}\right), \\
\partial_{\tilde{t}} \tilde{v} & =O(\Delta \tilde{t}), & \partial_{\tilde{t}} \tilde{E}+\tilde{\tau} \partial_{\tilde{x}}(\tilde{p} \tilde{u}) & =O(\Delta \tilde{t})+O(M \Delta \tilde{x}) .
\end{aligned}
$$

The rescaled discretization of the transport step is consistent with

$$
\partial_{\tilde{t}} \tilde{\varphi}+\tilde{u} \partial_{\tilde{x}} \tilde{\varphi}=O(\Delta \tilde{t})+O(\Delta \tilde{x})+O(M \Delta \tilde{x}),
$$

and the equivalent equation verified by the rescaled scheme reads

$$
\left\{\begin{aligned}
\partial_{\tilde{t}} \tilde{\rho}+\partial_{\tilde{x}}(\tilde{\rho} \tilde{u}) & =O(\Delta \tilde{t})+O(\Delta \tilde{x})+O(M \Delta \tilde{x}), \\
\partial_{\tilde{t}}(\tilde{\rho} \tilde{u})+\partial_{\tilde{x}}\left(\tilde{\rho} \tilde{u}^{2}\right)+\frac{1}{M^{2}} \partial_{\tilde{x}} \tilde{p} & =O(\Delta \tilde{t})+O(\Delta \tilde{x})+O(M \Delta \tilde{x})+O\left(\frac{\Delta \tilde{x}}{M}\right), \\
\partial_{\tilde{t}}(\tilde{\rho} \tilde{v})+\partial_{\tilde{x}}(\tilde{\rho} \tilde{u} \tilde{v}) & =O(\Delta \tilde{t})+O(\Delta \tilde{x})+O(M \Delta \tilde{x}), \\
\partial_{\tilde{t}}(\tilde{\rho} \tilde{E})+\partial_{\tilde{x}}[(\tilde{\rho} \tilde{E}+\tilde{p}) \tilde{u}] & =O(\Delta \tilde{t})+O(\Delta \tilde{x})+O(M \Delta \tilde{x}) .
\end{aligned}\right.
$$

Proof. There exists three smooth functions $A, B$ and $C$ of magnitude 1 with respect to $M$ such that

$$
\begin{aligned}
\tilde{u}_{j+1 / 2}^{*} & =\frac{\tilde{u}_{j+1}^{n}+\tilde{u}_{j}^{n}}{2}+M \Delta \tilde{x} A\left(x_{j+1 / 2}, t^{n}\right)+O\left(M \Delta \tilde{x}^{2}\right), \\
\tilde{\Pi}_{j+1 / 2}^{*} & =\frac{\tilde{\Pi}_{j+1}^{n}+\tilde{\Pi}_{j}^{n}}{2}+M \Delta \tilde{x} B\left(x_{i+1 / 2}, t^{n}\right)+O\left(M \Delta \tilde{x}^{2}\right), \\
\tilde{\Pi}_{j+1 / 2}^{*} \tilde{u}_{j+1 / 2}^{*} & =\frac{\left(\tilde{u}_{j+1}^{n}+\tilde{u}_{j}^{n}\right)\left(\tilde{\Pi}_{j+1}^{n}+\tilde{\Pi}_{j}^{n}\right)}{4}+M \Delta \tilde{x} C\left(x_{i+1 / 2}, t^{n}\right)+O\left(M \Delta \tilde{x}^{2}\right) .
\end{aligned}
$$


Injecting the above relation into $(21)$ we get

$$
\left\{\begin{aligned}
\tilde{L}_{j} \tilde{\rho}_{j}^{n+1-} & =\tilde{\rho}_{j}^{n}, \\
\tilde{L}_{j}(\tilde{\rho} \tilde{u})_{j}^{n+1-} & =(\tilde{\rho} \tilde{u})_{j}^{n}-\frac{\Delta \tilde{t}}{M^{2}} \frac{\tilde{\Pi}_{j+1}^{n}-\tilde{\Pi}_{j-1}^{n}}{2 \Delta \tilde{x}}+O\left(\frac{\Delta \tilde{x} \Delta \tilde{t}}{M}\right) \\
\tilde{L}_{j}\left(\tilde{\rho}_{j} \tilde{v}\right)^{n+1-} & =(\tilde{\rho} \tilde{v})_{j}^{n}, \\
L_{j}(\tilde{\rho} \tilde{E})_{j}^{n+1-} & =(\tilde{\rho} \tilde{E})_{j}^{n}-\Delta \tilde{t}\left(\frac{\left(\tilde{u}_{j+1}^{n}+\tilde{u}_{j}^{n}\right)\left(\tilde{\Pi}_{j+1}^{n}+\tilde{\Pi}_{j}^{n}\right)}{4 \Delta \tilde{x}}-\frac{\left(\tilde{u}_{j-1}^{n}+\tilde{u}_{j}^{n}\right)\left(\tilde{\Pi}_{j-1}^{n}+\tilde{\Pi}_{j}^{n}\right)}{4 \Delta \tilde{x}}\right)+O(M \Delta \tilde{x} \Delta \tilde{t}), \\
\tilde{L}_{j} & =1+\Delta \tilde{t} \frac{\tilde{u}_{j+1}^{n}-\tilde{u}_{j-1}^{n}}{2 \Delta \tilde{x}}+O(M \Delta \tilde{x} \Delta \tilde{t}) .
\end{aligned}\right.
$$

This yields

$$
\left\{\begin{aligned}
\tilde{L}_{j} \tilde{\rho}_{j}^{n+1-} & =\tilde{\rho}_{j}^{n}, \\
\tilde{L}_{j}(\tilde{\rho} \tilde{u})_{j}^{n+1-} & =(\tilde{\rho} \tilde{u})_{j}^{n}-\frac{\Delta \tilde{t}}{M^{2}} \partial_{\tilde{x}} \tilde{p}+O\left(\frac{\Delta \tilde{x} \Delta \tilde{t}}{M}\right)+O\left(\Delta \tilde{x}^{2} \Delta \tilde{t}\right), \\
\tilde{L}_{j}\left(\tilde{\rho}_{j} \tilde{v}\right)^{n+1-} & =(\tilde{\rho} \tilde{v})_{j}^{n}, \\
L_{j}(\tilde{\rho} \tilde{E})_{j}^{n+1-} & =(\tilde{\rho} \tilde{E})_{j}^{n}-\Delta \tilde{t} \partial_{\tilde{x}}(\tilde{p} \tilde{u})+O(M \Delta \tilde{x} \Delta \tilde{t})+O\left(\Delta \tilde{x}^{2} \Delta \tilde{t}\right), \\
\tilde{L}_{j} & =1+\Delta \tilde{t} \partial_{\tilde{x}} \tilde{u}+O(M \Delta \tilde{x} \Delta \tilde{t})+O\left(\Delta \tilde{x}^{2} \Delta \tilde{t}\right) .
\end{aligned}\right.
$$

Remark 1. For smooth solutions in the low Mach regime, we have $\partial_{\tilde{x} \tilde{x}} \tilde{p}=O\left(M^{2}\right)$. We used this relation to obtain the term $O\left(\Delta \tilde{x}^{2} \Delta \tilde{t}\right)$ in $27 \mathrm{~b}$.

Let us remark that (27) is indeed consistent at order 1 with respect to $\Delta x$ with

$$
\begin{aligned}
\partial_{\tilde{t}} \tilde{\tau}-\tilde{\tau} \partial_{\tilde{x}} \tilde{u} & =O(\Delta \tilde{t})+O(M \Delta \tilde{x}), & \partial_{\tilde{t}} \tilde{u}+\frac{\tilde{\tau}}{M^{2}} \partial_{\tilde{x}} \tilde{p} & =O(\Delta \tilde{t})+O\left(\frac{\Delta \tilde{x}}{M}\right), \\
\partial_{\tilde{t}} \tilde{v} & =O(\Delta \tilde{t}), & \partial_{\tilde{t}} \tilde{E}+\tilde{\tau} \partial_{\tilde{x}}(\tilde{p} \tilde{u}) & =O(\Delta \tilde{t})+O(M \Delta \tilde{x}) .
\end{aligned}
$$

Now we turn to the transport step. Accounting for the low Mach hypothesis, 22 becomes

$$
\frac{1}{\Delta \tilde{t}}\left(\tilde{\varphi}_{j}^{n+1}-\tilde{L}_{j} \tilde{\varphi}_{j}^{n+1-}\right)+\frac{1}{2 \Delta \tilde{x}}\left(\tilde{\varphi}_{j+1 / 2}^{n+1-}\left(\tilde{u}_{j+1}^{n}+\tilde{u}_{j}^{n}\right)-\tilde{\varphi}_{j-1 / 2}^{n+1-}\left(\tilde{u}_{j}^{n}+\tilde{u}_{j-1}^{n}\right)\right)=O(M \Delta \tilde{x}),
$$

hence

$$
\frac{1}{\Delta \tilde{t}}\left(\tilde{\varphi}_{j}^{n+1}-\tilde{L}_{j} \tilde{\varphi}_{j}^{n+1-}\right)+\partial_{\tilde{x}}(\tilde{\varphi} \tilde{u})=O(\Delta \tilde{x})+O(M \Delta \tilde{x}),
$$

which is consistent with $\partial_{\tilde{t}} \tilde{\varphi}+\tilde{u} \partial_{\tilde{x}} \tilde{\varphi}=O(\Delta \tilde{t})+O(\Delta \tilde{x})+O(M \Delta \tilde{x})$. Finally, using (27) into (28) we finally obtain the desired result.

Remark 2. It is important to note that the analysis we proposed in this section cannot be considered as an exhaustive explanation for the behavior of the numerical scheme in the Low Mach regime. It just merely provides magnitude estimate of the truncation error. Considering the same lines with additional hypotheses: $\tilde{\rho}, \tilde{u}, \tilde{v}, \tilde{E}$ are solution of the rescaled gas dynamics equations in the low Mach regime with well-prepared conditions [11], then one can show that the $O(\Delta \tilde{x} / M)$ term in (25b) does vanish [11] for one-dimensional problems set over the whole real line. The analysis is delicate and depends on many hypotheses: for two-dimensional problems same results can be obtained for discretization over a triangular mesh with periodic boundary conditions. However, this no longer works for two-dimensional Cartesian meshes where the classical Godunov-type solvers perform poorly with periodic boundary conditions. More general boundary conditions require a specific study for each case [11, 10].

\section{Low Mach correction}

The equivalent equation (25) satisfied by the rescaled scheme is clearly not satisfactory because of the term $O\left(\frac{\Delta \tilde{x}}{M}\right)$ which behaves bad when $M \ll \Delta \tilde{x}$. This suggests to modify the scheme accordingly. 


\subsection{Correction of the low Mach behavior: a simple flux modification}

In the light of the previous asymptotic analysis, we propose to leave the projection step unchanged and rather focus on the acoustic step of the scheme. In the acoustic step, we suggest to simply replace $\Pi_{j+1 / 2}^{*}$ by

$$
\Pi_{j+1 / 2}^{*, \theta}=\frac{1}{2}\left(\Pi_{j}^{n}+\Pi_{j+1}^{n}\right)-\theta_{j+1 / 2} \frac{a_{j+1 / 2}}{2}\left(u_{j+1}^{n}-u_{j}^{n}\right) .
$$

The associated dimensionless flux reads

$$
\tilde{\Pi}_{j+1 / 2}^{*, \theta}=\frac{1}{2}\left(\tilde{\Pi}_{j}^{n}+\tilde{\Pi}_{j+1}^{n}\right)-\theta_{j+1 / 2} \frac{\tilde{a}_{j+1 / 2} M}{2}\left(\tilde{u}_{j+1}^{n}-\tilde{u}_{j}^{n}\right) .
$$

This yields the following modified scheme for the acoustic step.

$$
\left\{\begin{aligned}
\mathbf{W}_{j}^{n+1-} & =\mathbf{W}_{j}^{n}-\frac{\Delta t}{\Delta x}\left(\mathbf{F}_{j+1 / 2}-\mathbf{F}_{j-1 / 2}\right), \\
\mathbf{F}_{j+1 / 2} & =\mathbf{F}^{\theta}\left(\mathbf{W}_{j}^{n}, \mathbf{W}_{j+1}^{n}\right), \\
\mathbf{F}^{\theta}\left(\mathbf{W}_{L}, \mathbf{W}_{R}\right) & =\left(-u^{*}, \Pi^{*, \theta}, 0, \Pi^{*, \theta} u^{*}, a^{2} u^{*}\right)^{T} .
\end{aligned}\right.
$$

Let us underline that this modification solely alters the non-centered terms of the pressure flux. In other words this does not modify the ultimate consistency of $\Pi_{j+1 / 2}^{*, \theta}$ with the pressure value, it does impact the numerical dissipation involved with the discretization of the pressure terms. This approach complies with several previous works that have been investigating the approximation of the low Mach regime like [18, 24, 14]. While such modification is usually delicate with regards to the stability of the numerical scheme, we will nevertheless see that the resulting modified numerical scheme is still endowed with stability properties (see section 5.3). In the sequel, in order to perform an equivalent equation analysis with the modified pressure flux, we consider a smooth function $x \mapsto \theta$ such that $\theta_{j+1 / 2}=\theta\left(x_{j+1 / 2}\right)$. We have the following consistency properties for the numerical scheme with the modified pressure flux $\Pi_{j+1 / 2}^{*, \theta}$.

Proposition 2. In the low Mach regime, the rescaled discretization (31) of the acoustic step is consistent with

$$
\begin{aligned}
\partial_{\tilde{t}} \tilde{\tau}-\tilde{\tau} \partial_{\tilde{x}} \tilde{u} & =O(\Delta \tilde{t})+O(M \Delta \tilde{x}), & \partial_{\tilde{t}} \tilde{u}+\frac{\tilde{\tau}}{M^{2}} \partial_{\tilde{x}} \tilde{p} & =O(\Delta \tilde{t})+O\left(\frac{\theta \Delta \tilde{x}}{M}\right), \\
\partial_{\tilde{t}} \tilde{v} & =O(\Delta \tilde{t}), & \partial_{\tilde{t}} \tilde{E}+\tilde{\tau} \partial_{\tilde{x}}(\tilde{p} \tilde{u}) & =O(\Delta \tilde{t})+O(M \Delta \tilde{x})+O(M \theta \Delta \tilde{x}) .
\end{aligned}
$$

The rescaled discretization of the transport step is consistent with

$$
\partial_{\tilde{t}} \tilde{\varphi}+\tilde{u} \partial_{\tilde{x}} \tilde{\varphi}=+O(\Delta \tilde{t})+O(\Delta \tilde{x})+O(M \Delta \tilde{x}),
$$

and the equivalent equation verified by the rescaled scheme reads

$$
\left\{\begin{aligned}
\partial_{\tilde{t}} \tilde{\rho}+\partial_{\tilde{x}}(\tilde{\rho} \tilde{u}) & =O(\Delta \tilde{t})+O(\Delta \tilde{x})+O(M \Delta \tilde{x}) \\
\partial_{\tilde{t}}(\tilde{\rho} \tilde{u})+\partial_{\tilde{x}}\left(\tilde{\rho} \tilde{u}^{2}\right) \frac{1}{M^{2}}+\partial_{\tilde{x}} \tilde{p} & =O(\Delta \tilde{t})+O(\Delta \tilde{x})+O\left(\frac{\theta \Delta \tilde{x}}{M}\right) \\
\partial_{\tilde{t}}(\tilde{\rho} \tilde{v})+\partial_{\tilde{x}}(\tilde{\rho} \tilde{u} \tilde{v}) & =O(\Delta \tilde{t})+O(\Delta \tilde{x})+O(M \Delta \tilde{x}) . \\
\partial_{\tilde{t}}(\tilde{\rho} \tilde{E})+\partial_{\tilde{x}}[(\tilde{\rho} \tilde{E}+\tilde{p}) \tilde{u}] & =O(\Delta \tilde{t})+O(\Delta \tilde{x})+O(M \Delta \tilde{x})+O(M \theta \Delta \tilde{x}) .
\end{aligned}\right.
$$

As a consequence, provided that we impose the asymptotic behavior $\theta_{j+1 / 2}=O(M)$, the truncation error is uniform with respect to $M$.

Proof. Following similar lines as in the proof of proposition 1 and using the same notations, there exists three smooth functions $A, B, C$ and $D$ of magnitude 1 with respect to $M$ such that

$$
\begin{aligned}
\tilde{u}_{j+1 / 2}^{*} & =\frac{\tilde{u}_{j+1}^{n}+\tilde{u}_{j}^{n}}{2}+M \Delta \tilde{x} A\left(x_{j+1 / 2}, t^{n}\right)+O\left(M \Delta \tilde{x}^{2}\right), \\
\tilde{\Pi}_{j+1 / 2}^{*, \theta} & =\frac{\tilde{p}_{j+1}^{n}+\tilde{p}_{j}^{n}}{2}+\theta_{j+1 / 2} M \Delta \tilde{x} B\left(x_{i+1 / 2}, t^{n}\right)+O\left(M \Delta \tilde{x}^{2}\right), \\
\tilde{\Pi}_{j+1 / 2}^{*, \theta} \tilde{u}_{j+1 / 2}^{*} & =\frac{\left(\tilde{u}_{j+1}^{n}+\tilde{u}_{j}^{n}\right)\left(\tilde{p}_{j+1}^{n}+\tilde{p}_{j}^{n}\right)}{4}+M \Delta \tilde{x} C\left(x_{i+1 / 2}, t^{n}\right)+M \theta_{j+1 / 2} \Delta \tilde{x} D\left(x_{i+1 / 2}, t^{n}\right)+O\left(M \Delta \tilde{x}^{2}\right) .
\end{aligned}
$$


The rest of the analysis follows the same line as the proof of proposition 1 Using 21] we get

$$
\left\{\begin{aligned}
\tilde{L}_{j} \tilde{\rho}_{j}^{n+1-} & =\tilde{\rho}_{j}^{n} \\
\tilde{L}_{j}(\tilde{\rho} \tilde{u})_{j}^{n+1-} & =(\tilde{\rho} \tilde{u})_{j}^{n}-\frac{\Delta \tilde{t}}{M^{2}} \partial_{\tilde{x}} \tilde{p}+O\left(\frac{\theta \Delta \tilde{x} \Delta \tilde{t}}{M}\right)+O\left(\Delta \tilde{x}^{2} \Delta \tilde{t}\right) \\
\tilde{L}_{j}\left(\tilde{\rho}_{j} \tilde{v}\right)^{n+1-} & =(\tilde{\rho} \tilde{v})_{j}^{n} \\
L_{j}(\tilde{\rho} \tilde{E})_{j}^{n+1-} & =(\tilde{\rho} \tilde{E})_{j}^{n}-\Delta \tilde{t} \partial_{\tilde{x}}(\tilde{p} \tilde{u})+O(M \Delta \tilde{x} \Delta \tilde{t})+O(M \theta \Delta \tilde{x} \Delta \tilde{t})+O\left(\Delta \tilde{x}^{2} \Delta \tilde{t}\right), \\
\tilde{L}_{j} & =1+\Delta \tilde{t} \partial_{\tilde{x}} \tilde{u}+O(M \Delta \tilde{x} \Delta \tilde{t})+O\left(\Delta \tilde{x}^{2} \Delta \tilde{t}\right) .
\end{aligned}\right.
$$

and 22 yields again

$$
\frac{1}{\Delta \tilde{t}}\left(\tilde{\varphi}_{j}^{n+1}-\tilde{L}_{j} \tilde{\varphi}_{j}^{n+1-}\right)+\partial_{\tilde{x}}(\tilde{\varphi} \tilde{u})=O(\Delta \tilde{x})+O(M \Delta \tilde{x}) .
$$

Relations (33) and (34) provides the desired results.

Remark 3. In the light of the truncation error that appears in 25), one can see that it is not necessary to involve a correction for the energy flux term in (31c). It would be possible to consider a numerical scheme with the definition (10a) for the velocity at the interface, the modified pressure (29) for interface pressure terms and $\Pi^{*} u^{*}$ for the energy flux.

\subsection{Approximate Riemann solver for the modified acoustic scheme}

The modified numerical scheme (31) for the acoustic step belongs to the category of flux-based solver. Indeed, this solver relies on an update formula (31a) that involves the modified flux (31c). We will prove in this section that this modified flux solver can also be obtained thanks to an approximate Riemann solver in the sense of Harten, Lax and van Leer [17, 1], see also Annex B for a quick refresh on this, that is consistent with the integral form of (8). This formalism is useful to establish stability properties. We have the following proposition.

Proposition 3. There exists a simple approximate Riemann solver that is an approximation of the Riemann problem associated with the relaxed acoustic problem (8) and whose associated flux matches the flux of the modified acoustic solver. More precisely, there exists a self-similar function

$$
\mathbf{W}_{R P}^{\theta}\left(\frac{m}{t} ; \mathbf{W}_{L}, \mathbf{W}_{R}\right)=(\tau, u, v, E, \Pi)\left(\frac{m}{t} ; \mathbf{W}_{L}, \mathbf{W}_{R}\right)= \begin{cases}\mathbf{W}_{L}, & \text { if } m / t<-a, \\ \mathbf{W}_{L}^{*, \theta}, & \text { if }-a \leq m / t<0 \\ \mathbf{W}_{R}^{*, \theta}, & \text { if } 0 \leq m / t<+a \\ \mathbf{W}_{R}, & \text { if }+a \leq m / t\end{cases}
$$

such that

$$
\mathbf{F}^{\theta}\left(\mathbf{W}_{R}, \mathbf{W}_{L}\right)=\mathbf{F}\left(\mathbf{W}_{L}\right)-\int_{-\infty}^{0}\left[\mathbf{W}_{R P}^{\theta}\left(\xi ; \mathbf{W}_{L}, \mathbf{W}_{R}\right)-\mathbf{W}_{L}\right] \mathrm{d} \xi=\mathbf{F}\left(\mathbf{W}_{R}\right)+\int_{0}^{+\infty}\left[\mathbf{W}_{R P}^{\theta}\left(\xi ; \mathbf{W}_{L}, \mathbf{W}_{R}\right)-\mathbf{W}_{R}\right] \mathrm{d} \xi
$$

The states $\mathbf{W}_{L}^{*, \theta}=\left(\tau_{L}^{*, \theta}, u_{L}^{*, \theta}, v_{L}^{*, \theta}, \Pi_{L}^{*, \theta}\right)^{T}$ and $\mathbf{W}_{R}^{*, \theta}=\left(\tau_{R}^{*, \theta}, u_{R}^{*, \theta}, v_{R}^{*, \theta}, \Pi_{R}^{*, \theta}\right)^{T}$ are given by

$$
\begin{aligned}
\tau_{L}^{*, \theta} & =\tau_{L}+\frac{1}{a}\left(u^{*}-u_{L}\right), & \tau_{R}^{*, \theta} & =\tau_{R}+\frac{1}{a}\left(u_{R}-u^{*}\right), \\
u_{L}^{*, \theta} & =u^{*}+\frac{1}{2}(\theta-1)\left(u_{R}-u_{L}\right), & u_{R}^{*, \theta} & =u^{*}+\frac{1}{2}(1-\theta)\left(u_{R}-u_{L}\right), \\
v_{L}^{*, \theta} & =v_{L}, & v_{R}^{*, \theta} & =v_{R}, \\
E_{L}^{*, \theta} & =E_{L}+\frac{1}{a}\left(\Pi_{L} u_{L}-\Pi^{*, \theta} u^{*}\right), & E_{R}^{*, \theta} & =E_{R}+\frac{1}{a}\left(\Pi^{*, \theta} u^{*}-\Pi_{R} u_{R}\right) \\
\Pi_{L}^{*, \theta} & =\Pi^{*}, & \Pi_{R}^{*, \theta} & =\Pi^{*} .
\end{aligned}
$$


Proof. Suppose that $\mathbf{W}_{\mathrm{RP}}^{\theta}$ is consistent with the integral form of the relaxed acoustic problem (8) then for a given $\mathbf{W}_{L}$ and $\mathbf{W}_{R}$ we have

$$
\mathbf{F}\left(\mathbf{W}_{R}\right)-\mathbf{F}\left(\mathbf{W}_{L}\right)=-a\left(\mathbf{W}_{L}^{*, \theta}-\mathbf{W}_{L}\right)+a\left(\mathbf{W}_{R}-\mathbf{W}_{R}^{*, \theta}\right),
$$

which reads

$$
\mathbf{W}_{R}^{*, \theta}+\mathbf{W}_{L}^{*, \theta}=\mathbf{W}_{R}+\mathbf{W}_{L}-\frac{1}{a}\left(\mathbf{F}\left(\mathbf{W}_{R}\right)-\mathbf{F}\left(\mathbf{W}_{L}\right)\right) .
$$

If the resulting flux of this approximate Riemann solver is $F^{\theta}\left(\mathbf{W}_{L}, \mathbf{W}_{R}\right)$ then 36 is verified and yields

$$
2 F^{\theta}\left(\mathbf{W}_{L}, \mathbf{W}_{R}\right)=\mathbf{F}\left(\mathbf{W}_{R}\right)+\mathbf{F}\left(\mathbf{W}_{L}\right)-a\left(\mathbf{W}_{L}^{*, \theta}-\mathbf{W}_{L}\right)-a\left(\mathbf{W}_{R}-\mathbf{W}_{R}^{*, \theta}\right)
$$

or equivalently

$$
\mathbf{W}_{R}^{*, \theta}-\mathbf{W}_{L}^{*, \theta}=\mathbf{W}_{R}-\mathbf{W}_{L}+\frac{1}{a}\left(2 F^{\theta}\left(\mathbf{W}_{L}, \mathbf{W}_{R}\right)-\mathbf{F}\left(\mathbf{W}_{L}\right)-\mathbf{F}\left(\mathbf{W}_{R}\right)\right) .
$$

Both (38) and (39) provide

$$
\mathbf{W}_{L}^{*, \theta}=\mathbf{W}_{L}-\frac{1}{a}\left(F^{\theta}\left(\mathbf{W}_{L}, \mathbf{W}_{R}\right)-\mathbf{F}\left(\mathbf{W}_{L}\right)\right), \quad \mathbf{W}_{R}^{*, \theta}=\mathbf{W}_{R}+\frac{1}{a}\left(F^{\theta}\left(\mathbf{W}_{L}, \mathbf{W}_{R}\right)-\mathbf{F}\left(\mathbf{W}_{R}\right)\right) .
$$

This yields the desired results.

Using this approximate Riemann solver, we can deduce that the modified acoustic solver (31) is stable under the same CFL conditions (17) that does not depend on the Mach number $M$. Moreover, when $\theta=1$ the self-similar function $\mathbf{W}_{R P}^{\theta}$ defined in proposition 3 degenerates to the exact solution of the Riemann problem associated with relaxed acoustic system (8).

Finally, if one takes into account the equilibrium projection step of the relaxation strategy into the approximate Riemann solver of proposition 3 we have $\Pi_{L}=p^{\operatorname{EOS}}\left(\tau_{L}, e_{L}\right)$, and $\Pi_{R}=p^{\mathrm{EOS}}\left(\tau_{R}, e_{R}\right)$. Under this assumption, it is easy to check that the first coordinates $(\tau, u, v, E)$ of the self similar function $\mathbf{W}_{\mathrm{RP}}^{\theta}$ are consistent with the integral form of the acoustic system (6).

\subsection{Properties of the modified operator splitting scheme}

We start this section by examining the ability of the modified operator splitting scheme to satisfies a discrete entropy inequality. In the sequel, $I\left(b, b^{\prime}\right) \subset \mathbb{R}$ will denote the interval whose bounds are $b \in \mathbb{R}$ and $b^{\prime} \in \mathbb{R}$. We consider the following slightly more restrictive subcharacteristic condition

$$
\begin{aligned}
\tau_{L}^{*}>0, & -\partial_{\tau} p^{\operatorname{EOS}}\left(\tau, s_{L}\right) \leq a^{2}, & \forall \tau \in I\left(\tau_{L}, \tau_{L}^{*}\right), \\
\tau_{R}^{*}>0, & -\partial_{\tau} p^{\operatorname{EOS}}\left(\tau, s_{R}\right) \leq a^{2}, & \forall \tau \in I\left(\tau_{R}, \tau_{R}^{*}\right),
\end{aligned}
$$

and we start with the two following technical results. we also refer the reader to Annex B for a quick refresh on this topic.

Lemma 1. Consider the solution of Riemann problem for the relaxed acoustic system (8). Suppose that (40) is verified. Let $s_{k}=s^{\mathrm{EOS}}\left(\tau_{k}, e_{k}\right), k=L, R$, we have

$$
e_{k}^{*}-e^{\operatorname{EOS}}\left(\tau_{k}^{*}, s_{k}\right)-\frac{\left(p^{\operatorname{EOS}}\left(\tau_{k}^{*}, s_{k}\right)-\Pi^{*}\right)^{2}}{2 a^{2}} \geq 0
$$

Proof. We consider the case $k=R$ and set for $\tau \in I\left(\tau_{R}, \tau_{R}^{*}\right)$

$$
\begin{aligned}
\phi(\tau)=e^{\mathrm{EOS}}\left(\tau, s_{R}\right)-\frac{p^{\mathrm{EOS}}\left(\tau, s_{R}\right)^{2}}{2 a^{2}}-e^{\mathrm{EOS}}\left(\tau_{R}^{*}, s_{R}\right) & +\frac{p^{\mathrm{EOS}}\left(\tau_{R}^{*}, s_{R}\right)^{2}}{2 a^{2}} \\
& +p^{\mathrm{EOS}}\left(\tau_{R}^{*}, s_{R}\right)\left(\tau+\frac{p^{\mathrm{EOS}}\left(\tau, s_{R}\right)}{a^{2}}-\tau_{R}^{*}-\frac{p^{\mathrm{EOS}}\left(\tau_{R}^{*}, s_{R}\right)}{a^{2}}\right) .
\end{aligned}
$$

We have $\phi^{\prime}(\tau)=\left(p^{\operatorname{EOS}}\left(\tau, s_{R}\right)-p^{\operatorname{EOS}}\left(\tau_{R}^{*}, s_{R}\right)\right)\left(1-\rho^{2} c^{2}\left(\tau, s_{R}\right) / a^{2}\right)$. If $\tau_{R}>\tau>\tau_{R}^{*}\left(\right.$ resp. $\left.\tau_{R}<\tau<\tau_{R}^{*}\right)$ the Weyl assumptions (2) provides $p^{\operatorname{EOS}}\left(\tau, s_{R}\right)-p^{\operatorname{EOS}}\left(\tau_{R}^{*}, s_{R}\right)<0\left(\operatorname{resp} . p^{\operatorname{EOS}}\left(\tau, s_{R}\right)-p^{\operatorname{EOS}}\left(\tau_{R}^{*}, s_{R}\right)>0\right)$ and together with hypothesis 40 this yields $\phi^{\prime}(\tau) \geq 0$ (resp. $\left.\phi^{\prime}(\tau) \leq 0\right)$. As $\phi\left(\tau_{R}^{*}\right)=0$ we obtain that $\phi\left(\tau_{R}\right)>\phi\left(\tau_{R}^{*}\right)=0$ for $\tau \in I\left(\tau_{R}, \tau_{R}^{*}\right)$. Using the Riemann invariant jump relation $\left(e_{R}^{*}-\frac{\Pi^{*}}{2 a^{2}}\right)=\left(e_{R}-\frac{\Pi_{R}}{2 a^{2}}\right)$, one obtains $0<\phi\left(\tau_{R}\right)=e_{R}^{*}-e^{\operatorname{EOS}}\left(\tau_{R}^{*}, s_{R}\right)-\frac{1}{2 a^{2}}\left(p^{\operatorname{EOS}}\left(\tau_{R}^{*}, s_{R}\right)-\Pi^{*}\right)^{2}$. The same lines applies for the case $k=L$. 
Lemma 2. Let $\theta \in \mathbb{R}$, and $e_{k}^{*, \theta}=E_{k}^{*, \theta}-\left(u_{k}^{*, \theta}\right)^{2} / 2$ for $k=L, R$ then we have

$$
e_{k}^{*, \theta}-e^{\operatorname{EOS}}\left(\tau_{k}^{*, \theta}, s_{k}\right)-\frac{1}{2 a^{2}}\left(p^{\operatorname{EOS}}\left(\tau_{k}^{*, \theta}, s_{k}\right)-\Pi^{*}\right)^{2}+\frac{(1-\theta)^{2}\left(u_{R}-u_{L}\right)^{2}}{8} \geq 0, \quad k=L, R .
$$

Proof. One has $u_{R}^{*, \theta}=u^{*}+(1-\theta)\left(u_{R}-u_{L}\right) / 2$ and $\Pi^{*, \theta}=\Pi^{*}+(1-\theta) a\left(u_{R}-u_{L}\right) / 2$ and together with (37) one obtains $e_{R}^{*, \theta}=e_{R}^{*}-(1-\theta)^{2}\left(u_{R}-u_{L}\right)^{2} / 8$. Injecting this relation into 41) and noticing that $\tau_{R}^{*, \theta}=\tau_{R}^{*}$ provides the desired result for $k=R$. The case $k=L$ is obtained with the same lines.

It is now clear that the inequalities

$$
-\frac{1}{2 a^{2}}\left(p^{\operatorname{EOS}}\left(\tau_{k}^{*, \theta}, s_{R}\right)-\Pi^{*}\right)^{2}+\frac{(1-\theta)^{2}\left(u_{R}-u_{L}\right)^{2}}{8} \leq 0, \quad k=L, R
$$

can help us equip the modified numerical scheme with a discrete entropy inequality.

Proposition 4. Let $s_{k}^{*, \theta}=s^{\mathrm{EOS}}\left(\tau_{k}^{*, \theta}, e_{k}^{*, \theta}\right)$ for $k=L, R$. If assumption (43) is verified, we have

$$
0 \leq-a\left(s_{L}^{*, \theta}-s_{L}\right)+a\left(s_{R}-s_{R}^{*, \theta}\right) .
$$

Inequality (44) implies that the modified scheme (31) for the acoustic step is consistent with the integral form of the entropy inequality

$$
\partial_{t} s(\tau, e) \leq 0
$$

Moreover, the explicit modified scheme (31) is equipped with a discrete entropy inequality. Indeed there exists a numerical flux function $q_{j+1 / 2}^{n}=q\left(\mathbf{W}_{j}^{n}, \mathbf{W}_{j+1}^{n}\right)$ that is consistent with 0 when $\Delta t$ and $\Delta x$ tend to 0 such that

$$
s\left(\tau_{j}^{n+1-}, e_{j}^{n+1-}\right)-s\left(\tau_{j}^{n}, e_{j}^{n}\right)+\tau_{j}^{n} \frac{\Delta t}{\Delta x}\left(q_{j+1 / 2}^{n}-q_{j-1 / 2}^{n}\right) \leq 0 .
$$

Proof. Let $k=L, R$, under hypothesis (43), we have that $e_{k}^{*, \theta} \geq e^{\operatorname{EOS}}\left(\tau_{k}^{*, \theta}, s_{k}\right)$. According to (2) $\epsilon \mapsto$ $s^{\operatorname{EOS}}\left(\tau_{k}^{*, \theta}, \epsilon\right)$ is increasing, thus $s^{\operatorname{EOS}}\left(\tau_{k}^{*, \theta}, e_{k}^{*, \theta}\right)=s_{k}^{*, \theta} \geq s^{\operatorname{EOS}}\left(\tau_{k}^{*, \theta}, e^{\operatorname{EOS}}\left(\tau_{k}^{*, \theta}, s_{k}\right)\right)=s_{k}$. Inequality (44) follows trivially. Relation (44) expresses the consistency with the integral form of (45) and it provides the entropy inequality (46) (see [1, Chap. 2] and Annex B).

We can now state the following entropic property for the full modified operator splitting explicit scheme composed by (31) and (14).

Proposition 5. If the assumptions (43), (17) and (18) are verified, then the explicit scheme defined by (31) and (14) verifies the following discrete entropy inequality

$$
\rho_{j}^{n+1} s\left(\tau_{j}^{n+1}, e_{j}^{n+1}\right)-\rho_{j}^{n} s\left(\tau_{j}^{n}, e_{j}^{n}\right)+\frac{\Delta t}{\Delta x}\left(g_{j+1 / 2}^{n}-g_{j-1 / 2}^{n}\right) \leq 0,
$$

where the numerical entropy flux is defined by

$$
g_{j+1 / 2}^{n}=\left(u_{j+1 / 2}^{*}\right)^{+} \rho_{j}^{n+1-} s\left(\tau_{j}^{n+1-}, e_{j}^{n+1-}\right)+\left(u_{j+1 / 2}^{*}\right)^{-} \rho_{j}^{n+1-} s\left(\tau_{j+1}^{n+1-}, e_{j+1}^{n+1-}\right)+q_{j+1 / 2}^{n} .
$$

Proof. Let $\phi \in(\rho, \rho u, \rho v, \rho E)$, under the CFL assumption (18) the transport scheme 14) expresses $\phi_{j}^{n+1}$ as a convex combination of $\phi_{i}^{n+1-}, i=j-1, j, j+1$, indeed one has

$$
\phi_{j}^{n+1}=\frac{\Delta t}{\Delta x}\left(u_{j-1 / 2}^{*}\right)^{+} \phi_{j-1}^{n+1-}+\left(1-\frac{\Delta t}{\Delta x}\left(\left(u_{j+1 / 2}^{*}\right)^{-}-\left(u_{j-1 / 2}^{*}\right)^{+}\right)\right) \phi_{j}^{n+1-}+\frac{\Delta t}{\Delta x}\left(u_{j+1 / 2}^{*}\right)^{-} \phi_{j+1}^{n+1-} .
$$

As the mapping $(\rho, \rho u, \rho v, \rho E) \mapsto-(\rho s)(\tau, e)$ is a strictly convex function (see for example [15]) we obtain that

$$
\begin{aligned}
-(\rho s)\left(\tau_{j}^{n+1}, e_{j}^{n+1}\right) & \leq-\frac{\Delta t}{\Delta x}\left(u_{j-1 / 2}^{*}\right)^{+}(\rho s)\left(\tau_{j-1}^{n+1-}, e_{j-1}^{n+1-}\right) \\
- & \left(1-\frac{\Delta t}{\Delta x}\left(\left(u_{j+1 / 2}^{*}\right)^{-}-\left(u_{j-1 / 2}^{*}\right)^{+}\right)\right)(\rho s)\left(\tau_{j}^{n+1-}, e_{j}^{n+1-}\right)-\frac{\Delta t}{\Delta x}\left(u_{j+1 / 2}^{*}\right)^{-}(\rho s)\left(\tau_{j-1}^{n+1-}, e_{j+1}^{n+1-}\right) .
\end{aligned}
$$

Using relation 46 one obtains 47. 
We now sum up the main properties of the modified operator splitting scheme.

Theorem 1. Suppose that (17), 18) (12) are satisfied, the explicit scheme defined by (31) and (14) verifies

1. the scheme is conservative with respect to the density $\rho$, the momentum $\rho u$ and total energy $\rho E$,

2. the density $\rho_{j}^{n}$ is positive for all $j$ and $n>0$ provided that $\rho_{j}^{0}$ is positive for all $j$,

3. if $\theta=\mathcal{O}(M)$, then the truncation error of the numerical scheme is uniform with respect to $M<1$,

4. if (43) is verified then the numerical scheme is equipped with a discrete entropy inequality,

5. if 433 is verified then $e_{j}^{n}>0$ for all $j \in \mathbb{Z}$ and all $n \in \mathbb{N}$.

It is clear from (32b) that the choice $\theta=O(M)$ is natural for the modified scheme to have an equivalent equation which is satisfactory when $M \ll \Delta \tilde{x}$ (uniform consistency w.r.t. $M$ ). At this stage $\theta=O(M)$ is not made precise, see section 6 below. Let us now discuss the new condition which is related to the correction $\theta$.

\subsubsection{Behavior of condition $\sqrt{43}$ in the low-Mach regime for a perfect gas equation of state}

We have just seen that the scheme is entropic provided that $\sqrt{43}$ is satisfied. In this section, we study the compatibility in the low Mach regime between the condition (43) that is required to obtain a discrete entropy inequality and the condition $\theta=O(M)$ that is required to have uniform consistency with respect to $M$ (see section 5). If $\left|u_{R}-u_{L}\right|=0$, any value of $\theta \in \mathbb{R}$ verifies condition (43), we can then assume that $\left|u_{R}-u_{L}\right|>0$. We consider the case of a Perfect Gas EOS defined by $p^{\mathrm{EOS}}(\rho, e)=(\gamma-1) \rho e$, where $\gamma$ is the specific heat ratio. First, let us recall that $\tau_{k}^{*, \theta}=\tau_{k}^{*}$ and $\Pi_{k}=p^{\mathrm{EOS}}\left(\tau_{k}, s_{k}\right), k=R, L$. For $k=R$, relation 43 reads

$$
|1-\theta| \leq \frac{2}{a} \frac{\left|p^{\operatorname{EOS}}\left(\tau_{R}^{*}, s_{R}\right)-\Pi^{*}\right|}{\left|u_{R}-u_{L}\right|} .
$$

Let us remark that the right hand side of this inequality does not depend on $\theta$. The Perfect Gas assumption provides that $p^{\mathrm{EOS}}\left(\tau_{R}^{*}, s_{R}\right)=\Pi_{R}\left(\tau_{R} / \tau_{R}^{*}\right)^{\gamma}$, therefore thanks to the definition of $\Pi^{*}$ we get

$$
p^{\mathrm{EOS}}\left(\tau_{R}^{*}, s_{R}\right)-\Pi^{*}=\Pi_{R}\left(\tau_{R} / \tau_{R}^{*}\right)^{\gamma}-\frac{\Pi_{L}+\Pi_{R}}{2}+\frac{a}{2}\left(u_{R}-u_{L}\right) .
$$

The definition of $\tau_{R}^{*, \theta}=\tau_{R}^{*}$ by (37) using the dimensionless parameters defined by 19 gives $\tilde{\tau}_{R}^{*}=\tilde{\tau}_{R}+$ $\left(\tilde{\Pi}_{R}-\tilde{\Pi}_{L}\right) /\left(2 \tilde{a}^{2}\right)+M\left(\tilde{u}_{R}-\tilde{u}_{L}\right) /(2 \tilde{a})$. If one now supposes that the flow is locally in the low Mach regime, then we have $\partial_{\tilde{x}} \tilde{\Pi}=O\left(M^{2}\right)$, therefore $\Pi_{R}-\Pi_{L}=O\left(M^{2} \Delta \tilde{x}\right)$. Thus we obtain

$$
\frac{\tilde{\tau}_{R}^{*}}{\tilde{\tau}_{R}}=1+M \frac{\tilde{u}_{R}-\tilde{u}_{L}}{2 \tilde{a} \tilde{\tau}_{R}}+O\left(M^{2} \Delta \tilde{x}\right) .
$$

Injecting the above relation into 50, , we obtain

$$
\frac{p^{\mathrm{EOS}}\left(\tau_{R}^{*}, s_{R}\right)-\Pi^{*}}{p_{0}}=-\frac{M}{2}\left[1-\frac{\gamma \tilde{\Pi}_{R}}{\tilde{a}^{2} \tilde{\tau}_{R}}\right] \tilde{a}\left(\tilde{u}_{R}-\tilde{u}_{L}\right)+O\left(M^{2} \Delta \tilde{x}\right) .
$$

Using the fact that $\gamma p^{\operatorname{EOS}}\left(\tau_{R}, s_{R}\right)=\gamma \Pi_{R}=\rho_{R}\left(c_{R}\right)^{2}$ for a Perfect Gas in the previous relation allows to recast 49 into

$$
|1-\theta| \leq\left|1-\left(\frac{\tilde{\rho}_{R} \tilde{c}_{R}}{\tilde{a}}\right)^{2}+O\left(\frac{M \Delta \tilde{x}}{\left|\tilde{u}_{R}-\tilde{u}_{L}\right|}\right)\right| .
$$

Let us recall that by definition : $\tilde{a}=K \max \left(\tilde{\rho}_{R} \tilde{c}_{R}, \tilde{\rho}_{L} \tilde{c}_{L}\right)$ with $K \geq 1$. Suppose without loss of generality that $\tilde{\rho}_{R} \tilde{c}_{R}=\max \left(\tilde{\rho}_{R} \tilde{c}_{R}, \tilde{\rho}_{L} \tilde{c}_{L}\right)$ then $\left(\tilde{\rho}_{R} \tilde{c}_{R}\right)^{2} / \tilde{a}^{2}=1 / K$ and the condition (51) becomes

$$
|1-\theta| \leq\left|1-\left(\frac{1}{K}\right)^{2}+O\left(\frac{M \Delta \tilde{x}}{\left|\tilde{u}_{R}-\tilde{u}_{L}\right|}\right)\right|
$$




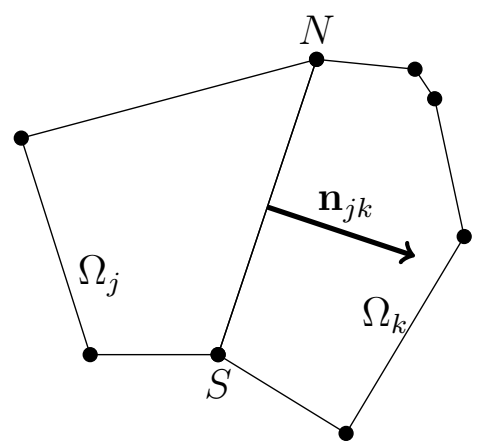

Figure 1: the face $\Gamma_{j k}=\overline{\Omega_{j}} \cap \overline{\Omega_{k}}$ defined the segment $(N S)$ has a unit normal vector $\mathbf{n}_{j k}$ oriented from $\Omega_{j}$ to $\Omega_{k}$.

Behavior when $M \rightarrow 0$. When $M \rightarrow 0$ the above inequality yields that $\theta \geq(1 / K)^{2}$ if one wants to enforce uniform consistency with respect to $M$ by setting $\theta=O(M)$. tThis leads to a contradiction. As a conclusion, a correction scheme with $\theta=O(M)$ does not provide an entropic scheme in the asymptotic limit $M \rightarrow 0$. On the contrary, $\theta=1$ which correspond to the classic unmodified scheme is still entropic. Nevertheless, it is reasonable to consider that in the limit $M \rightarrow 0$, the solution of the gas dynamics equation is smooth and therefore the consistency with an entropy criterion is a less critical matter.

\subsection{Extension to several space dimensions with unstructured grids}

Without loss of generality, we suppose that $\Omega \subset \mathbb{R}^{2}$ is a polygonal domain that is covered by a set of $N$ polygonal cells $\left(\Omega_{j}\right)_{1 \leq j \leq N}$. Let $\Gamma$ be a face of a cell $\Omega_{j}, 1 \leq j \leq N$. If $\Gamma \subset \partial \Omega$, we suppose that there exists a single $k>N$ that will help to index ghost values for boundary conditions and we shall note $\Gamma=\Gamma j k$. If $\Gamma \cap \partial \Omega=\emptyset$, we suppose that the mesh is admissible in the sense that there exists a single $1 \leq k \leq N$ such that $\Gamma=\overline{\Omega_{j}} \cap \overline{\Omega_{k}}$. Moreover, for $1 \leq j \leq N$ and $1 \leq k \leq N$ we suppose that $\overline{\Omega_{i}} \cap \overline{\Omega_{j}}$ can either be empty, a vertex or a single face of the mesh. If $\Gamma_{j k}$ be the face of a cell $\Omega_{j}$ then $\mathbf{n}_{j k}$ will denote the unit vector normal to $\Gamma_{j k}$ pointing out of $\Omega_{j}$. We define $N(j)$ the set of indices $k$ such that $\Gamma_{j k}$ is a face of $\Omega_{j}$. Let $E=\{(j, k) \mid 1 \leq j, k \leq N, k \in N(j)\}$ and $E^{\text {ext }}=\left\{(j, k) \mid 1 \leq j \leq N, k \in N(j), \Gamma_{j k} \subset \partial \Omega\right\}$. In sequel $x=\left(x_{1}, x_{2}\right) \in \mathbb{R}^{2}$ will denote the space variable.

We will now present a natural extension of our discretization strategy for the case of multi-dimensional problems with unstructured grids. Within this framework, the classical Lagrange-Remap algorithm involves tracking a genuine multi-dimensional moving mesh. This task is a very delicate matter as the mesh may be dramatically distorted during the simulation. We will here present a much simpler approach that relies on the alternative guideline proposed in section 3. A similar approach was used to derive an explicit scheme for two-component interface problems in [?].

Consider the operator splitting of (1) into the following systems

$$
\left\{\begin{array}{r}
\partial_{t} \rho+\rho \operatorname{div}(\mathbf{u})=0 \\
\partial_{t}(\rho \mathbf{u})+\rho \mathbf{u} \operatorname{div}(\mathbf{u})+\nabla p=0 \\
\partial_{t}(\rho E)+\rho E \operatorname{div}(\mathbf{u})+\operatorname{div}(P \mathbf{u})=0
\end{array}\right.
$$

and

$$
\left\{\begin{aligned}
\partial_{t} \rho+(\mathbf{u} \cdot \nabla) \rho & =0, \\
\partial_{t}(\rho \mathbf{u})+(\mathbf{u} \cdot \nabla) \rho \mathbf{u} & =0, \\
\partial_{t}(\rho E)+(\mathbf{u} \cdot \nabla) \rho E & =0 .
\end{aligned}\right.
$$

Before going any further, let us note that we obtain similar properties as for the systems (4) and (5). Indeed system (52) is a quasilinear hyperbolic system that involves the two nonlinear acoustic waves of velocity $\pm c$ and two null velocity contact discontinuities waves. System (52) only involves acoustic phenomena while freezing the material transport, while $(53)$ is pure multi-dimensional transport system at the material velocity $\mathbf{u}$.

We adopt the same strategy as in section 3 given a fluid state $(\rho, \rho \mathbf{u}, \rho E)_{j}^{n}$, 
- update the fluid state to the value $(\rho, \rho \mathbf{u}, \rho E)_{j}^{n+1-}$ by approximating the solution of 52 ,

- update the fluid state to the value $(\rho, \rho \mathbf{u}, \rho E)_{j}^{n+1}$ by approximating the solution of $[53$.

Approximation of the acoustic system 52 ,

System 52 can be expressed

$$
\partial_{t} \tau-\tau(x, t) \operatorname{div}(\mathbf{u})=0, \quad \partial_{t} \mathbf{u}+\tau(x, t) \nabla p=0, \quad E_{t}+\tau(x, t) \operatorname{div}(p \mathbf{u})=0 .
$$

Using the same lines as in section 3 we consider a Suliciu-type relaxation approximation

$$
\partial_{t} \tau-\tau(x, t) \operatorname{div}(\mathbf{u})=0, \quad \partial_{t} \mathbf{u}+\tau(x, t) \nabla \Pi=0, \quad E_{t}+\tau(x, t) \operatorname{div}(p \mathbf{u})=0, \quad \Pi_{t}+\tau(x, t) a^{2} \operatorname{div}(\mathbf{u})=\nu(p-\Pi),
$$

in the regime $\nu \rightarrow+\infty$. Once again, for $t \in\left[t^{n}, t^{n}+\Delta t\right)$, this task is achieved by setting $\Pi\left(x, t^{n}\right)=p\left(x, t^{n}\right)$ and then solving the relaxation system for $\nu=0$. We approximate again $\tau(x, t) \partial_{x_{r}}$ by $\tau\left(x, t^{n}\right) \partial_{x_{r}}$ for $r=1,2$ when $t \in\left[t^{n}, t^{n}+\Delta t\right)$. In the regime $\lambda=0$ our approximation of (52) becomes

$$
\partial_{t} \tau-\tau\left(x, t^{n}\right) \operatorname{div}(\mathbf{u})=0, \quad \partial_{t} \mathbf{u}+\tau\left(x, t^{n}\right) \nabla \Pi=0, \quad E_{t}+\tau\left(x, t^{n}\right) \operatorname{div}(p \mathbf{u})=0, \quad \Pi_{t}+\tau\left(x, t^{n}\right) a^{2} \operatorname{div}(\mathbf{u})=0 .
$$

If $b$ is a flow parameter and $b_{j}^{n}$ is an approximation of $\frac{1}{\left|\Omega_{j}\right|} \int_{\Omega_{j}} b\left(x, t^{n}\right) \mathrm{d} x$, we solve (54) thanks to the following classical Finite-Volume method

$$
\left\{\begin{array}{l}
\mathbf{u}_{j}^{n+1-}=\mathbf{u}_{j}^{n}-\tau_{j}^{n} \Delta t \sum_{k \in N(j)} \sigma_{j k} \Pi_{j k}^{* \theta} \mathbf{n}_{j k}, \\
\Pi_{j}^{n+1-}=\Pi_{j}^{n}-\tau_{j}^{n} \Delta t \sum_{k \in N(j)} \sigma_{j k}\left(a_{j k}\right)^{2} u_{j k}^{*}, \\
\tau_{j}^{n+1-}=\tau_{j}^{n}+\tau_{j}^{n} \Delta t \sum_{k \in N(j)} \sigma_{j k} u_{j k}^{*}, \\
E_{j}^{n+1-}=E_{j}^{n}-\tau_{j}^{n} \Delta t \sum_{k \in N(j)} \sigma_{j k} \Pi_{j k}^{*, \theta} u_{j k}^{*},
\end{array}\right.
$$

where $\sigma_{j k}=\left|\Gamma_{j k}\right| /\left|\Omega_{j}\right|$.

The three scalar quantities $a_{j k}, \Pi_{j k}^{*, \theta}$ and $u_{j k}^{*}$ that respectively represent an average sound velocity, a pressure and the normal velocity at the face $\Gamma_{j k}$. In order to define these quantities, we classicaly take advantage of the fact that (54) is rotational invariant. This allows to associate in the referential of each face $\Gamma_{j k}$ a Suliciu-type relaxation approximation of a one-dimensional Riemann problem in the frame of the face. Noting $\sharp \in\{n, n+1-\}$, this leads us to set

$$
\begin{aligned}
a_{j k} & \geq \max \left[(\rho c)_{j}^{n},(\rho c)_{k}^{n}\right], \\
u_{j k}^{*} & =\frac{1}{2} \mathbf{n}_{j k}^{T}\left(\mathbf{u}_{j}^{\sharp}+\mathbf{u}_{k}^{\sharp}\right)-\frac{1}{2 a_{j k}}\left(\Pi_{k}^{\sharp}-\Pi_{j}^{\sharp}\right), \\
\Pi_{j k}^{*, \theta} & =\frac{1}{2}\left(\Pi_{j}^{\sharp}+\Pi_{k}^{\sharp}\right)-\frac{a_{j k} \theta_{j k}}{2} \mathbf{n}_{j k}^{T}\left(\mathbf{u}_{k}^{\sharp}-\mathbf{u}_{j}^{\sharp}\right) .
\end{aligned}
$$

When $\sharp=n$ the solver is explicit and when $\sharp=n+1-$, the solver is implicit.

Approximation of the transport system 53

In order to approximate the solution of (53), we simply use an upwind Finite-Volume scheme. Let $\varphi \in$ $\left\{\rho, \rho u_{1}, \rho u_{2}, \rho E\right\}$, we set

$$
\varphi_{j}^{n+1}=\varphi_{j}^{n+1-}-\Delta t \sum_{k \in N(j)} \sigma_{j k} u_{j k}^{*} \varphi_{j k}^{n+1-}+\Delta t \varphi_{j}^{n+1-} \sum_{k \in N(j)} \sigma_{j k} u_{j k}^{*},
$$

where $\varphi_{j k}^{n+1-}$ is defined by the upwind choice with respect to the sign of $u_{j k}^{*}$, namely

$$
\varphi_{j k}^{n+1-}= \begin{cases}\varphi_{j}^{n+1-}, & \text { if } u_{j k}^{*}>0, \\ \varphi_{k}^{n+1-}, & \text { if } u_{j k}^{*} \leq 0 .\end{cases}
$$


Proposition 6. The overall numerical scheme composed by the discretization steps (55a)-(55d) and (57) is conservative with respect to the variable $\rho$, $\rho \mathbf{u}$ and $\rho E$, for both the implicit solver and the explicit solver. The update of these variables from $t^{n}$ to $t^{n+1}$ reads

$$
\begin{aligned}
\rho_{j}^{n+1}-\rho_{j}^{n}+\Delta t \sum_{k \in N(j)} \sigma_{j k} \rho_{j k}^{n+1-} u_{j k}^{*} & =0, \\
(\rho \mathbf{u})_{j}^{n+1}-(\rho \mathbf{u})_{j}^{n}+\Delta t \sum_{k \in N(j)} \sigma_{j k}\left((\rho \mathbf{u})_{j k}^{n+1-} u_{j k}^{*}+\Pi_{j k}^{*, \theta} \mathbf{n}_{j k}\right) & =0, \\
(\rho E)_{j}^{n+1}-(\rho E)_{j}^{n}+\Delta t \sum_{k \in N(j)} \sigma_{j k}\left((\rho E)_{j k}^{n+1-}+\Pi_{j k}^{*, \theta}\right) u_{j k}^{*} & =0 .
\end{aligned}
$$

The semi-implicit solver obtained for $\sharp=n+1-$ can be decomposed along the following steps: the acoustic step first involves solving the linear system (55a)- $55 \mathrm{~b}$ ) for computing the acoustic velocity $\mathbf{u}_{j}^{n+1-}$ and pressure term $\Pi_{j}^{n+1-}$. The acoustic step is completed by the update of $\tau_{j}^{n+1-}$ and $E_{j}^{n+1-}$ thanks to the explicit procedures $(55 \mathrm{c})$ and $(55 \mathrm{~d})$. The last stage of the semi-implicit solver is achieved thanks to the explicit transport scheme (57).

We want now to investigate further the implicit system involved with the semi-implicit method for the specific case of wall-boundary conditions that we implement by imposing ghost values $\Pi_{k}^{n+1-}$ and $\mathbf{n}_{j k}^{T} \mathbf{u}_{k}^{n+1-}$ for a boundary face $\Gamma_{j k} \subset \partial \Omega$, where $1 \leq j \leq N$ and $k \in N(j), k>N$ with

$$
\Pi_{k}^{n+1-}=\Pi_{j}^{n+1-}, \quad \mathbf{n}_{j k}^{T} \mathbf{u}_{k}^{n+1-}=-\mathbf{n}_{j k}^{T} \mathbf{u}_{j}^{n+1-} .
$$

We have the following proposition.

Proposition 7. We consider the case of the semi-implicit solver with implementation of wall boundary conditions (59) and a uniform choice of a, i.e. $a_{j k}=a$ for all $1 \leq j \leq N$ and $k \in N(j)$. If $\tau_{j}^{n}>0$ for all $1 \leq j \leq N$, then the linear system (55a)-(55b) always possesses a single solution for any $\Delta t>0$ and $\theta_{j k}>0$.

Proof. For the sake of readability, we shall note here $\mathbf{u}_{j}^{n+1-}=\mathbf{u}_{j}$ and $\Pi_{j}^{n+1-}=\Pi_{j}$. The finite-dimension linear system (55a)-(55b) reads

$$
\left\{\begin{array}{c}
\left|\Omega_{j}\right| \mathbf{u}_{j}+\tau_{j}^{n} \Delta t \sum_{k \in N(j)}\left|\Gamma_{j k}\right|\left[\frac{1}{2}\left(\Pi_{j}+\Pi_{k}\right)-\frac{a \theta_{j k}}{2} \mathbf{n}_{j k}^{T}\left(\mathbf{u}_{k}-\mathbf{u}_{j}\right)\right] \mathbf{n}_{j k}=\left|\Omega_{j}\right| \mathbf{u}_{j}^{n}, \\
\left|\Omega_{j}\right| \Pi_{j}+\tau_{j}^{n} \Delta t \sum_{k \in N(j)}\left|\Gamma_{j k}\right| a^{2}\left[\frac{1}{2} \mathbf{n}_{j k}^{T}\left(\mathbf{u}_{j}+\mathbf{u}_{k}\right)-\frac{1}{2 a}\left(\Pi_{k}-\Pi_{j}\right)\right]=\left|\Omega_{j}\right| \Pi_{j}^{n} .
\end{array}\right.
$$

This system admits a unique solution if and only if $\mathbf{u}_{j}=0, \Pi_{j}=0,1 \leq j \leq N$ is the only solution of the particular case obtained for $\mathbf{u}_{j}^{n}=0, \Pi_{j}^{n}=0,1 \leq j \leq N$. Thus, let us now suppose that the right members of 60 are null, we proceed using an energy estimate type proof. Left multiply 60 a by $\frac{2 \mathbf{u}_{j}^{T}}{\tau_{j}^{n} \Delta t}$ and sum over $j$, we obtain

$$
0=\sum_{j=1}^{N} \frac{2\left|\Omega_{j}\right|\left|\mathbf{u}_{j}\right|^{2}}{\tau_{j}^{n} \Delta t}+\sum_{j=1}^{N} \sum_{k \in N(j)}\left|\Gamma_{j k}\right|\left(\Pi_{j}+\Pi_{k}\right)\left(\mathbf{u}_{j}^{T} \mathbf{n}_{j k}\right)-\sum_{j=1}^{N} \sum_{k \in N(j)}\left|\Gamma_{j k}\right| a \theta_{j k}\left(\mathbf{u}_{j}^{T} \mathbf{n}_{j k}\right)\left(\mathbf{u}_{k}-\mathbf{u}_{j}\right)^{T} \mathbf{n}_{j k} .
$$

Accounting for the fact that $\sum_{k \in N(j)}\left|\Gamma_{j k}\right| \mathbf{n}_{j k}=\mathbf{0}$, the second term of 61 verifies

$$
\sum_{j=1}^{N} \sum_{k \in N(j)}\left|\Gamma_{j k}\right|\left(\Pi_{j}+\Pi_{k}\right)\left(\mathbf{u}_{j}^{T} \mathbf{n}_{j k}\right)=\sum_{j=1}^{N} \sum_{k \in N(j)}\left|\Gamma_{j k}\right| \Pi_{k} \mathbf{u}_{j}^{T} \mathbf{n}_{j k}
$$


Using boundary conditions (59), the third term of 61) reads

$$
\begin{aligned}
\sum_{j=1}^{N} & \sum_{k \in N(j)}\left|\Gamma_{j k}\right| a \theta_{j k}\left(\mathbf{u}_{j}^{T} \mathbf{n}_{j k}\right)\left(\mathbf{u}_{k}-\mathbf{u}_{j}\right)^{T} \mathbf{n}_{j k} \\
\quad= & \sum_{(j, k) \in E}\left|\Gamma_{j k}\right| a \theta_{j k}\left[\left(\mathbf{u}_{j}^{T} \mathbf{n}_{j k}\right)\left(\mathbf{u}_{k}-\mathbf{u}_{j}\right)^{T} \mathbf{n}_{j k}+\left(\mathbf{u}_{k}^{T} \mathbf{n}_{k j}\right)\left(\mathbf{u}_{j}-\mathbf{u}_{k}\right)^{T} \mathbf{n}_{k j}\right]+\sum_{(j, k) \in E^{\mathrm{ext}}}\left|\Gamma_{j k}\right| a \theta_{j k}\left(\mathbf{u}_{j}^{T} \mathbf{n}_{j k}\right)\left(\mathbf{u}_{k}-\mathbf{u}_{j}\right)^{T} \mathbf{n}_{j k} \\
\quad= & -\sum_{(j, k) \in E}\left|\Gamma_{j k}\right| a \theta_{j k}\left[\left(\mathbf{u}_{k}-\mathbf{u}_{j}\right)^{T} \mathbf{n}_{j k}\right]^{2}-2 \sum_{(j, k) \in E^{\text {ext }}}\left|\Gamma_{j k}\right| a \theta_{j k}\left[\left(\mathbf{u}_{j}^{T} \mathbf{n}_{j k}\right)\right]^{2} .
\end{aligned}
$$

Finally we see that (61) is equivalent to

$$
0=\sum_{j=1}^{N} \frac{2\left|\Omega_{j}\right|\left|\mathbf{u}_{j}\right|^{2}}{\tau_{j}^{n} \Delta t}+\sum_{j=1}^{N} \sum_{k \in N(j)}\left|\Gamma_{j k}\right| \Pi_{k} \mathbf{u}_{j}^{T} \mathbf{n}_{j k}+\sum_{(j, k) \in E}\left|\Gamma_{j k}\right| a \theta_{j k}\left[\left(\mathbf{u}_{k}-\mathbf{u}_{j}\right)^{T} \mathbf{n}_{j k}\right]^{2}+2 \sum_{(j, k) \in E^{\text {ext }}}\left|\Gamma_{j k}\right| a \theta_{j k}\left[\left(\mathbf{u}_{j}^{T} \mathbf{n}_{j k}\right)\right]^{2} .
$$

Let us turn to the pressure equation $60 \mathrm{~b}$, we multiply by $\frac{2 \Pi_{j}}{\tau_{j}^{n} a^{2} \Delta t}$ and sum over all $1 \leq j \leq N$, this yields

$$
0=\sum_{j=1}^{N} \frac{2\left|\Omega_{j}\right| \Pi_{j}^{2}}{\tau_{j}^{n} a^{2} \Delta t}+\sum_{j=1}^{N} \sum_{k \in N(j)}\left|\Gamma_{j k}\right| \mathbf{n}_{j k}^{T}\left(\mathbf{u}_{j}+\mathbf{u}_{k}\right) \Pi_{j}-\sum_{j=1}^{N} \sum_{k \in N(j)} \frac{1}{a}\left|\Gamma_{j k}\right|\left(\Pi_{k}-\Pi_{j}\right) \Pi_{j} .
$$

Using once again $\sum_{k \in N(j)}\left|\Gamma_{j k}\right| \mathbf{n}_{j k}=\mathbf{0}$, we have for the second term of 63 that

$$
\sum_{j=1}^{N} \sum_{k \in N(j)}\left|\Gamma_{j k}\right| \mathbf{n}_{j k}^{T}\left(\mathbf{u}_{j}+\mathbf{u}_{k}\right) \Pi_{j}=\sum_{j=1}^{N} \sum_{k \in N(j)}\left|\Gamma_{j k}\right| \mathbf{n}_{j k}^{T} \mathbf{u}_{k} \Pi_{j} .
$$

Accounting for (59), the third term of 63 verifies

$$
\begin{aligned}
\sum_{j=1}^{N} \sum_{k \in N(j)} \frac{\left|\Gamma_{j k}\right|}{a}\left(\Pi_{k}-\Pi_{j}\right) \Pi_{j} & =\frac{1}{a} \sum_{(j, k) \in E}\left|\Gamma_{j k}\right|\left[\left(\Pi_{k}-\Pi_{j}\right) \Pi_{j}-\left(\Pi_{j}-\Pi_{k}\right) \Pi_{k}\right]+\frac{1}{a} \sum_{(j, k) \in E^{\text {ext }}}\left|\Gamma_{j k}\right|\left(\Pi_{k}-\Pi_{j}\right) \Pi_{j} \\
& =-\frac{1}{a} \sum_{(j, k) \in E}\left|\Gamma_{j k}\right|\left(\Pi_{k}-\Pi_{j}\right)^{2} .
\end{aligned}
$$

Then, we see that 63 also reads

$$
0=\sum_{j=1}^{N} \frac{2\left|\Omega_{j}\right| \Pi_{j}^{2}}{\tau_{j}^{n} a^{2} \Delta t}+\sum_{j=1}^{N} \sum_{k \in N(j)}\left|\Gamma_{j k}\right| \mathbf{n}_{j k}^{T} \mathbf{u}_{k} \Pi_{j}+\frac{1}{a} \sum_{(j, k) \in E}\left|\Gamma_{j k}\right|\left(\Pi_{k}-\Pi_{j}\right)^{2} .
$$

We now remark that

$$
\begin{aligned}
\sum_{j=1}^{N} \sum_{k \in N(j)}\left|\Gamma_{j k}\right| \mathbf{n}_{j k}^{T} \mathbf{u}_{k} \Pi_{j} & =\sum_{(j, k) \in E}\left|\Gamma_{j k}\right|\left(\mathbf{n}_{j k}^{T} \mathbf{u}_{k} \Pi_{j}+\mathbf{n}_{k j}^{T} \mathbf{u}_{j} \Pi_{k}\right)+\sum_{(j, k) \in E^{\text {ext }}}\left|\Gamma_{j k}\right| \mathbf{n}_{j k}^{T} \mathbf{u}_{k} \Pi_{j} \\
& =\sum_{(j, k) \in E}\left|\Gamma_{j k}\right| \mathbf{n}_{j k}^{T}\left(\mathbf{u}_{k} \Pi_{j}-\mathbf{u}_{j} \Pi_{k}\right)-\sum_{(j, k) \in E^{\text {ext }}}\left|\Gamma_{j k}\right| \mathbf{n}_{j k}^{T} \mathbf{u}_{j} \Pi_{j},
\end{aligned}
$$

and also that

$$
\begin{aligned}
\sum_{j=1}^{N} \sum_{k \in N(j)}\left|\Gamma_{j k}\right| \Pi_{k} \mathbf{u}_{j}^{T} \mathbf{n}_{j k} & =\sum_{(j, k) \in E}\left|\Gamma_{j k}\right|\left(\Pi_{k} \mathbf{u}_{j}^{T} \mathbf{n}_{j k}+\Pi_{j} \mathbf{u}_{k}^{T} \mathbf{n}_{k j}\right)+\sum_{(j, k) \in E^{\mathrm{ext}}}\left|\Gamma_{j k}\right| \Pi_{k} \mathbf{u}_{j}^{T} \mathbf{n}_{j k} \\
& =\sum_{(j, k) \in E}\left|\Gamma_{j k}\right| \mathbf{n}_{j k}^{T}\left(\Pi_{k} \mathbf{u}_{j}-\Pi_{j} \mathbf{u}_{k}\right)+\sum_{(j, k) \in E^{\mathrm{ext}}}\left|\Gamma_{j k}\right| \Pi_{j} \mathbf{n}_{j k}^{T} \mathbf{u}_{j} .
\end{aligned}
$$


Therefore

$$
\sum_{j=1}^{N} \sum_{k \in N(j)}\left|\Gamma_{j k}\right| \Pi_{j} \mathbf{u}_{k}^{T} \mathbf{n}_{j k}+\sum_{j=1}^{N} \sum_{k \in N(j)}\left|\Gamma_{j k}\right| \Pi_{k} \mathbf{u}_{j}^{T} \mathbf{n}_{j k}=0 .
$$

Thus, summing 62 and 64 , we obtain

$0=\sum_{j=1}^{N} \frac{2\left|\Omega_{j}\right|}{\tau_{j}^{n} \Delta t}\left(\left|\mathbf{u}_{j}\right|^{2}+\frac{\Pi_{j}^{2}}{a^{2}}\right)+\sum_{(j, k) \in E}\left|\Gamma_{j k}\right|\left\{a \theta_{j k}\left[\left(\mathbf{u}_{k}-\mathbf{u}_{j}\right)^{T} \mathbf{n}_{j k}\right]^{2}+\frac{\left(\Pi_{k}-\Pi_{j}\right)^{2}}{a}\right\}+2 \sum_{(j, k) \in E^{\text {ext }}}\left|\Gamma_{j k}\right|\left\{a \theta_{j k}\left[\left(\mathbf{u}_{j}^{T} \mathbf{n}_{j k}\right)\right]^{2}\right\}$.

This implies that $\left|\mathbf{u}_{j}\right|=\Pi_{j}=0$ for all $1 \leq j \leq N$.

Remark 4. It is possible to derive a similar proof for the case of periodic boundary conditions.

We now examine the stability of the multi-dimensional operator splitting strategy (55), (56) and (57). The acoustic step 55 in the explicit cases $\sharp=n$ is stable under the CFL condition

$$
\Delta t \max _{1 \leq j \leq N}\left[\tau_{j}^{n}\left(\max _{k \in N(j)} \sigma_{j k} a_{j k}\right)\right] \leq \frac{1}{2} .
$$

For both the explicit scheme $\sharp=n$ and semi-implicit scheme $\sharp=n+1-$, the transport step (57) is stable under the CFL condition

$$
\Delta t \max _{1 \leq j \leq N}\left(\sum_{k \in N(j)}\left|\sigma_{j k}\left(\mathbf{n}_{j k}^{T} \mathbf{u}_{j k}^{*, \theta}\right)\right|\right) \leq 1 .
$$

When one uses the semi-implicit scheme $\sharp=n+1-$, the condition 660 becomes implicit as the computation of $\mathbf{u}_{j k}^{*, \theta}$ depends on a given $\Delta t$. In our simulations with the semi-implicit scheme, we chose to compute $\Delta t$ thanks to the CFL condition (66) with the value $\mathbf{u}_{j k}^{*, \theta}$ given by the fully explicit scheme $\sharp=n$. It is then possible to check a posteriori that this $\Delta t$ value matches 66 .

We gather thereafter the properties of the explicit and semi-implicit multi-dimensional schemes.

Theorem 2. Suppose that (65), (66) and (12) are satisfied. The explicit scheme defined by (55) and (57) with $\sharp=n$ verifies

1. the scheme is conservative with respect to the density $\rho$, the momentum $\rho u$ and total energy $\rho E$,

2. the density $\rho_{j}^{n}$ is positive for all $j$ and $n>0$ provided that $\rho_{j}^{0}$ is positive for all $j$,

3. if $\theta=O(M)$, then the truncation error of the numerical scheme is uniform with respect to $M<1$,

4. if 443 is verified then the numerical scheme is equipped with a discrete entropy inequality,

5. if 433 is verified then $e_{j}^{n}>0$ for all $j \in \mathbb{Z}$ and all $n \in \mathbb{N}$.

Theorem 3. Suppose that (66) and (12) are satisfied. The semi-implicit scheme defined by (55) and (57) with $\sharp=n+1-$ verifies

1. the scheme is conservative with respect to the density $\rho$, the momentum $\rho u$ and total energy $\rho E$,

2. the density $\rho_{j}^{n}$ is positive for all $j$ and $n>0$ provided that $\rho_{j}^{0}$ is positive for all $j$,

3. if $\theta=O(M)$, then the truncation error of the numerical scheme is uniform with respect to $M<1$.

Let us note that the implicit treatment of the acoustic step leads to a CFL restriction (66) based only on (slow) material waves. 


\section{$6 \quad$ Numerical results}

In this section, we present numerical results computed thanks to the general operator splitting strategy (55), (56) and (57) with the following schemes:

- $\operatorname{EX}(\theta=1)$ : the explicit operator splitting scheme obtained for $\theta_{j k}=1$ and $\sharp=n$,

- $\operatorname{EX}(\theta=O(M))$ : the explicit modified operator splitting scheme obtained with the low Mach correction $\theta_{j k}=\min \left(\left|u_{j k}^{*}\right| / \max \left(c_{j}^{n}, c_{k}^{n}\right), 1\right)$ and $\sharp=n$,

- $\operatorname{EX}(\theta=0)$ : the explicit modified operator splitting scheme with centered pressure gradient $\theta_{j k}=0$ and $\sharp=n$,

- $\operatorname{IMEX}(\theta=1)$ : the semi-implicit operator splitting scheme with $\theta_{j k}=1$ and $\sharp=n+1-$,

- $\operatorname{IMEX}(\theta=O(M))$ : the modified semi-implicit operator splitting scheme with $\sharp=n+1-$ and a low Mach correction $\theta_{j k}$ defined as in the case of $\operatorname{EX}(\theta=O(M))$,

- $\operatorname{IMEX}(\theta=0)$ : the modified semi-implicit operator splitting scheme with a centered pressure gradient $\theta_{i j}=0$ and $\sharp=n+1-$.

Remark 5. The choice of the modification $\theta_{j k}=\min \left(\frac{\left|u_{j k}^{*}\right|}{\max \left(c_{j}^{n}, c_{k}^{n}\right)}, 1\right)$ corresponds to a low Mach correction. Indeed, this choice is non-dimensional, in $(0,1)$, such that $\theta=O(M)$ in the low Mach regime and $\theta=1$ for large Mach numbers. In this latter case, we then recover the classical scheme without modification.

In the sequel, we shall consider that the fluid follows a perfect gas equation of state $p=(\gamma-1) \rho e$ with a specific heat ratio $\gamma=1.4$. We will test schemes on both low Mach and order 1 Mach number test cases.

\subsection{Low Mach number examples}

In this section we will consider low Mach tests and try to examine two questions: the accuracy gain for simulations on coarse grid in the low Mach regime thanks to the proposed correction, then the benefit of using a semi-implicit strategy in term of CPU time.

\section{Vortex in a Box}

We consider a test performed in [5]. The computational domain is $\Omega=[0,1]^{2}$ with an initial condition is given by

$$
\begin{array}{ll}
\rho_{0}\left(x_{1}, x_{2}\right)=1-\frac{1}{2} \tanh \left(x_{2}-\frac{1}{2}\right), & u_{0}\left(x_{1}, x_{2}\right)=2 \sin ^{2}\left(\pi x_{1}\right) \sin \left(\pi x_{2}\right) \cos \left(\pi x_{2}\right), \\
p_{0}\left(x_{1}, x_{2}\right)=1000, & v_{0}\left(x_{1}, x_{2}\right)=-2 \sin \left(\pi x_{1}\right) \cos \left(\pi x_{1}\right) \sin ^{2}\left(\pi x_{2}\right) .
\end{array}
$$

No-slip boundary conditions are imposed on the domain boundaries. The Mach number for the resulting flows is of order 0.026, so that we are in the low Mach regime. Results are displayed in table 1 and figures 2 and 3 .

We first use the schemes $\operatorname{EX}(\theta=1)$ with a $400 \times 400$-cell and a $50 \times 50$-cell mesh. As expected the scheme performs poorly on the coarse mesh and the gain of accuracy is obvious when one refines the mesh: a mesh size of order $M$ is required, but it comes at a much higher price in terms of CPU time as we can see on table 1 . The $\operatorname{EX}(\theta=O(M))$ scheme gives good results even with the coarse $50 \times 50$-cell grid. With the low Mach correction scheme the connection between the accuracy of the solution and the mesh size does not seem to be constrained by $M$. Therefore, for a given target accuracy on a relatively coarse mesh, this numerical scheme is also much cheaper in term of CPU time.

Let us now turn to the semi-implicit strategies where the time step was chosen in agreement with the material CFL condition 66). While the $\operatorname{IMEX}(\theta=1)$ is not CPU intensive on a coarse mesh the results are very altered by the numerical diffusion. The $\operatorname{IMEX}(\theta=O(M))$ scheme performs fast and allows to recover numerical results that are as good as $\operatorname{EX}(\theta=O(M))$. As with the $\operatorname{EX}(\theta=M)$ scheme the accuracy seems much less constrained by the Mach number when it comes to choosing the time step and the mesh size. As we can see in table 1 . the $\operatorname{IMEX}(\theta=O(M))$ scheme is 3.34 times faster than the $\operatorname{EX}(\theta=O(M))$. 
Table 1: Vortex in a box test case. Comparison of the number of iterations and CPU time of $\operatorname{EX}(\theta=1)$, $\operatorname{EX}(\theta=O(M)), \operatorname{IMEX}(\theta=1)$ and $\operatorname{IMEX}(\theta=O(M))$ schemes to obtain solutions of figure 2 and figure 3 .

\begin{tabular}{cccccc}
\hline Numerical scheme & $\operatorname{EX}(\theta=1)$ & $\operatorname{EX}(\theta=1)$ & $\operatorname{EX}(\theta=O(M))$ & $\operatorname{IMEX}(\theta=1)$ & $\operatorname{IMEX}(\theta=O(M))$ \\
Mesh & $400 \times 400$ & $50 \times 50$ & $50 \times 50$ & $50 \times 50$ & $50 \times 50$ \\
\hline Number of iterations & 18457 & 2306 & 2305 & 43 & 56 \\
CPU time (s) & $9263.04(2 \mathrm{~h} 34 \mathrm{~min})$ & 17.14 & 19.3 & 3.75 & 5.77 \\
\hline
\end{tabular}

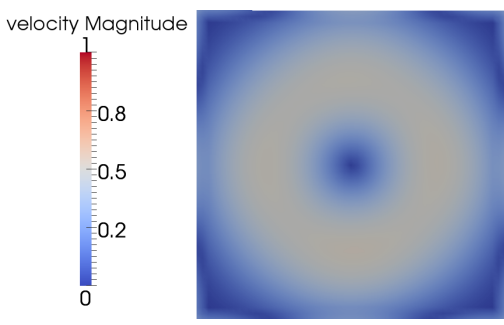

(a)

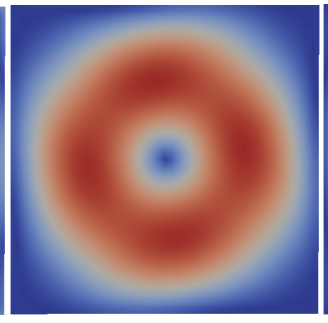

(b)

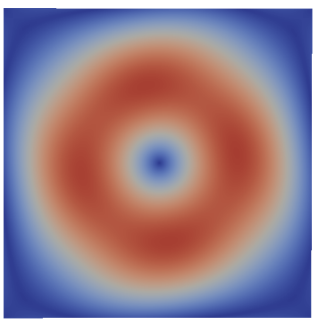

(c)

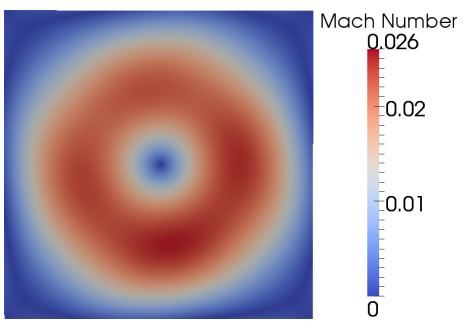

(d)

Figure 2: Vortex in a box test case with a Cartesian mesh. Profile at time $t=0.125 \mathrm{~s}$ of the velocity magnitude for (a) $\operatorname{EX}(\theta=1)$, (b) $\operatorname{EX}(\theta=O(M))$ with a $50 \times 50$-cell Cartesian mesh, (c) velocity magnitude obtained with $\operatorname{EX}(\theta=1)$ using a $400 \times 400$ Cartesian mesh and $(d)$ Mach number obtained with $\operatorname{EX}(\theta=1)$ using a $400 \times 400$ Cartesian mesh.
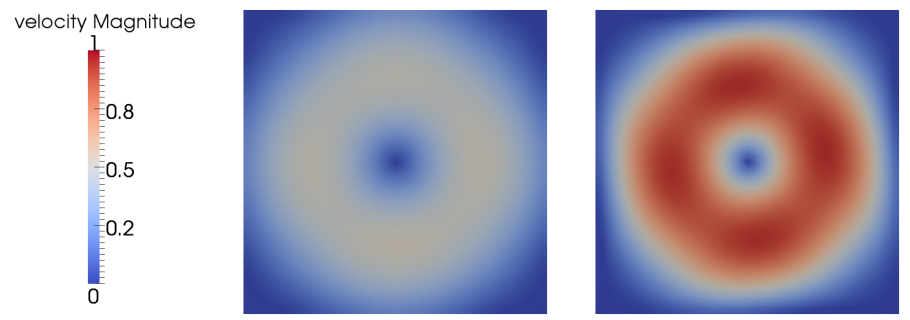

Figure 3: Vortex in a box test case with a Cartesian mesh. Profile at time $t=0.125 \mathrm{~s}$ of the velocity magnitude for the $\operatorname{IMEX}(\theta=1)$ scheme (left) and the $\operatorname{IMEX}(\theta=O(M))$ scheme (right) on a $50 \times 50$-cell Cartesian mesh. 
Table 2: Backward facing step test case. Comparison of the number of iterations and $\mathrm{CPU}$ time of $\operatorname{EX}(\theta=1)$, $\operatorname{EX}(\theta=O(M)), \operatorname{IMEX}(\theta=1)$ and $\operatorname{IMEX}(\theta=O(M))$ schemes to obtain solutions of figure 4 and figure 6

\begin{tabular}{ccccc}
\hline Numerical scheme & $\operatorname{EX}(\theta=1)$ & $\operatorname{EX}(\theta=O(M))$ & $\operatorname{IMEX}(\theta=1)$ & $\operatorname{IMEX}(\theta=O(M))$ \\
\hline Number of time steps & 5258803 & 5258784 & 4384 & 4970 \\
CPU time (s) & $62805.6(17 \mathrm{~h} \mathrm{27min})$ & $69764.7(19 \mathrm{~h} 22 \mathrm{~min})$ & $418.37(6 \mathrm{~min} 58 \mathrm{~s})$ & $500.83(8 \mathrm{~min} 20 \mathrm{~s})$ \\
\hline
\end{tabular}

\section{Backward facing step}

We consider now the case of an inviscid flow passing a backward facing step as derived from [7. The computational domain is $\Omega=[0,18] \times[0,2] \backslash(0,4) \times(0,1)$. The initial condition is given by

$$
\rho_{0}\left(x_{1}, x_{2}\right)=10, \quad u_{0}\left(x_{1}, x_{2}\right)=1, \quad p_{0}\left(x_{1}, x_{2}\right)=10^{5}, \quad v_{0}\left(x_{1}, x_{2}\right)=0 .
$$

We impose an inlet boundary condition at $\{0\} \times[1,2]$ and an outlet boundary condition at $\{12\} \times[0,2]$. Wall boundary conditions are set on other boundaries. This configuration leads to a low Mach flow with the order of magnitude $10^{-3} \leq M \leq 10^{-2}$. All tests are performed with a $220 \times 20$ Cartesian space grid.

Figure 4 and 6 display the flow profile at $t=50 \mathrm{~s}$, we observe that $\operatorname{EX}(\theta=1)$ and $\operatorname{IMEX}(\theta=1)$ schemes do not capture the vortex of the fluid in the low Mach velocity region. On the contrary, thanks to the low Mach correction $\operatorname{EX}(\theta=O(M))$ and $\operatorname{IMEX}(\theta=O(M))$ schemes are both able to capture this vortex with a coarse Cartesian Mesh. In term of CPU cost, measure are presented in table 2. We observe that the $\operatorname{IMEX}(\theta=O(M))$ scheme is 139.29 times faster than the $\operatorname{EX}(\theta=O(M))$ scheme thanks to the implicit treatment of the acoustic step, thanks to the use of material velocity CFL condition (66).

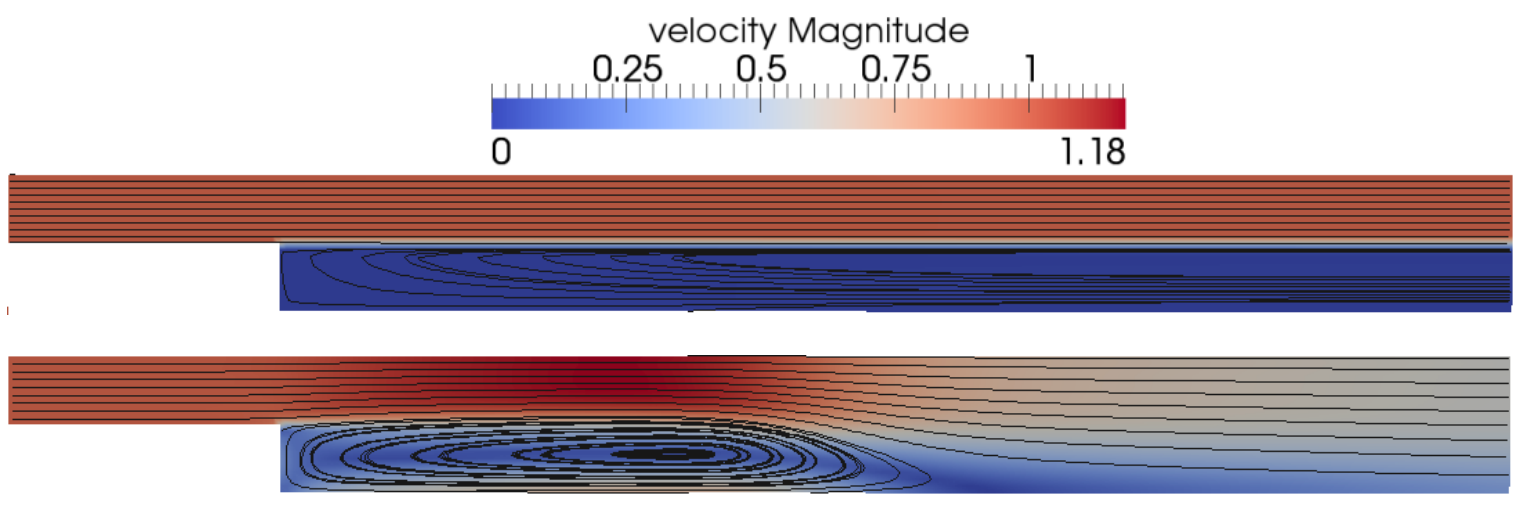

Figure 4: Backward facing step test case. Profile at $t=50 \mathrm{~s}$ of the velocity magnitude and stream lines for the $\operatorname{EX}(\theta=1)$ scheme (top) and the $\operatorname{EX}(\theta=O(M))$ scheme (bottom) on a $220 \times 20$-cell Cartesian mesh.

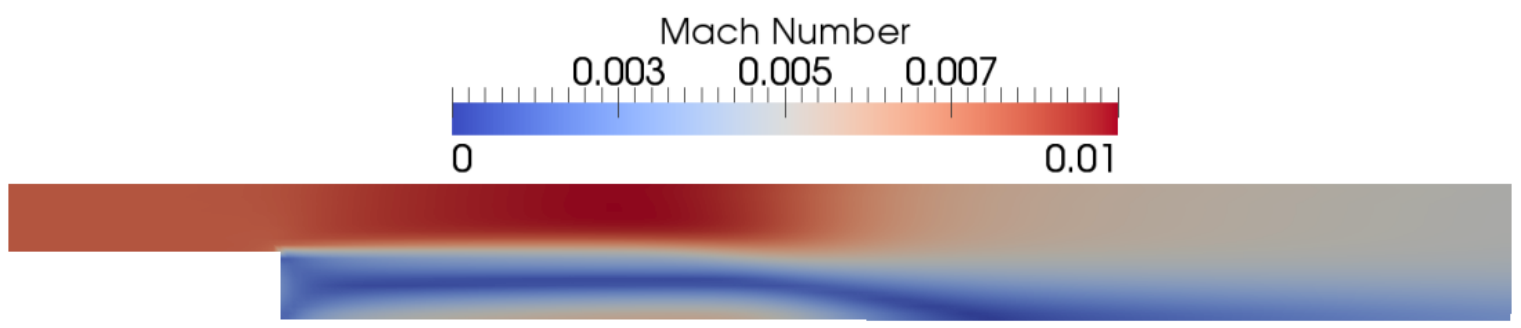

Figure 5: Backward facing step test case. Mapping at $t=50 \mathrm{~s}$ of the Mach number values for $\operatorname{EX}(\theta=O(M))$ scheme with a $220 \times 20$-cell Cartesian mesh. 

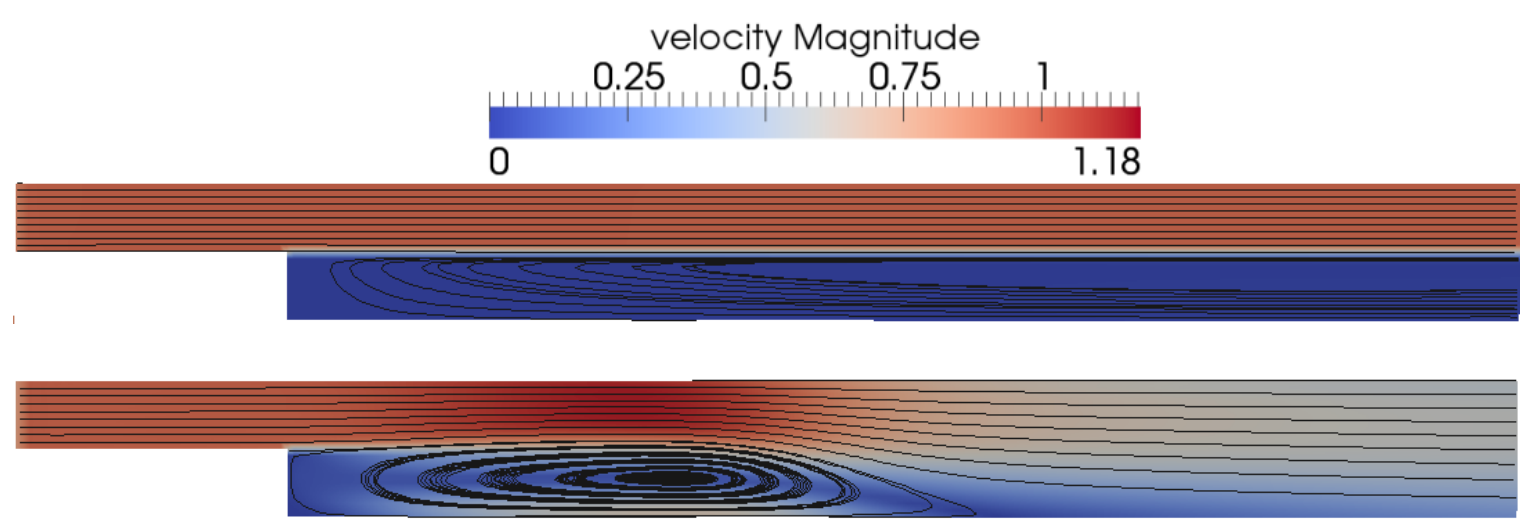

Figure 6: Backward facing step test case. Mapping at $t=50 \mathrm{~s}$ of the velocity magnitude and stream lines obtained with the $\operatorname{IMEX}(\theta=1)$ scheme (top) and the $\operatorname{IMEX}(\theta=O(M))$ scheme (bottom) using a $220 \times 20$-cell Cartesian mesh.

\subsection{Compressible flow examples}

In this section, we assess the ability of our operator splitting scheme to handle cases where the flow may not remain uniformly in the same Mach regime over the whole computational domain $\Omega$. We will see that even with a centred pressure discretization (which corresponds to the choice $\theta=0$ ), the solution remains stable but may be less precise in area where the Mach number is of order 1. The semi-implicit scheme becomes slower than the explicit scheme when the Mach number is of order 1 as the benefit from using a material CFL 66 condition instead of an acoustic CFL (65) becomes less beneficial but requires solving a linear system.

\section{D Sod shock tube}

We consider a variant of the classical Sod shock tube [26, that consists in solving the one-dimensional Riemann problem over $\Omega=[0,1]$ defined by the initial conditions $(\rho, u, P)=\left(1.0,0.0,10^{5}\right)$ for $x<0.5$ and $(\rho, u, P)=$ $\left(0.1,0.0,10^{4}\right)$ for $x>0.5$. We impose Neumann boundary conditions during the test. The domain is discretized over a 1000-cell grid. This resulting Mach number verifies $0<M<0.95$, so that we have both low Mach and order 1 Mach values. We plot the solution at $t=3.1 \times 10^{-4} \mathrm{~s}$.

Figure 7 displays the results obtained with $\operatorname{EX}(\theta)$ and $\operatorname{IMEX}(\theta)$ for $\theta=1$ and $\theta=0$. We use as reference solution an approximation computed with $\operatorname{EX}(\theta=1)$ using a 10000 -cell mesh. All schemes show a good agreement with the reference solution. The schemes $\operatorname{EX}(\theta=0)$ and $\operatorname{IMEX}(\theta=0)$ schemes are slightly less diffused than the $\operatorname{EX}(\theta=1)$ and $\operatorname{IMEX}(\theta=1)$ schemes results. Let us underline that despite part of the solutions clearly do not belong to the low Mach regime since $M \simeq 0.95$, the schemes $\operatorname{EX}(\theta=0)$ and $\operatorname{IMEX}(\theta=0)$ are stable and provide good numerical results while involving a centered pressure discretization with $\theta_{i j}=0$.

\section{D-Riemann problem}

We consider a 2D Riemann problem that consists of 4 shock waves [22]. We consider the domain $\Omega=[0,1]^{2}$. The initial condition is

$$
\left(\rho, u_{1}, u_{2}, P\right)\left(x_{1}, x_{2}, t=0\right)=\left\{\begin{array}{lll}
(0.1380,1.206,1.206,0.029), & \text { for } x_{1}<0.5, & x_{2}<0.5 \\
(0.5323,0.000,1.206,0.300), & \text { for } x_{1}>0.5, & x_{2}<0.5 \\
(0.5323,1.206,0.000,0.300), & \text { for } x_{1}<0.5, & x_{2}>0.5 \\
(1.5000,0.000,0.000,1.500), & \text { for } x_{1}>0.5, & x_{2}>0.5
\end{array}\right.
$$

We impose Neumann boundary conditions. This configuration leads to a Mach number that ranges from $10^{-5}$ to 3.15, i.e. according to the regions of the computation domain, the flow belongs to the low Mach regime or the order 1 Mach regime. We consider as a reference solution the approximation obtained with $\operatorname{EX}(\theta=1)$ for a $200 \times 200$-cell Cartesian mesh. Figures 8,910 and 11 display the result at $t=0.4 \mathrm{~s}$.

We observe in figure 8 and figure 9 that $\operatorname{EX}(\theta=0)$ and $\operatorname{IMEX}(\theta=0)$ schemes are stable for this test case with both low Mach and order 1 Mach number values regions. Both figure show that the wave pattern at the 


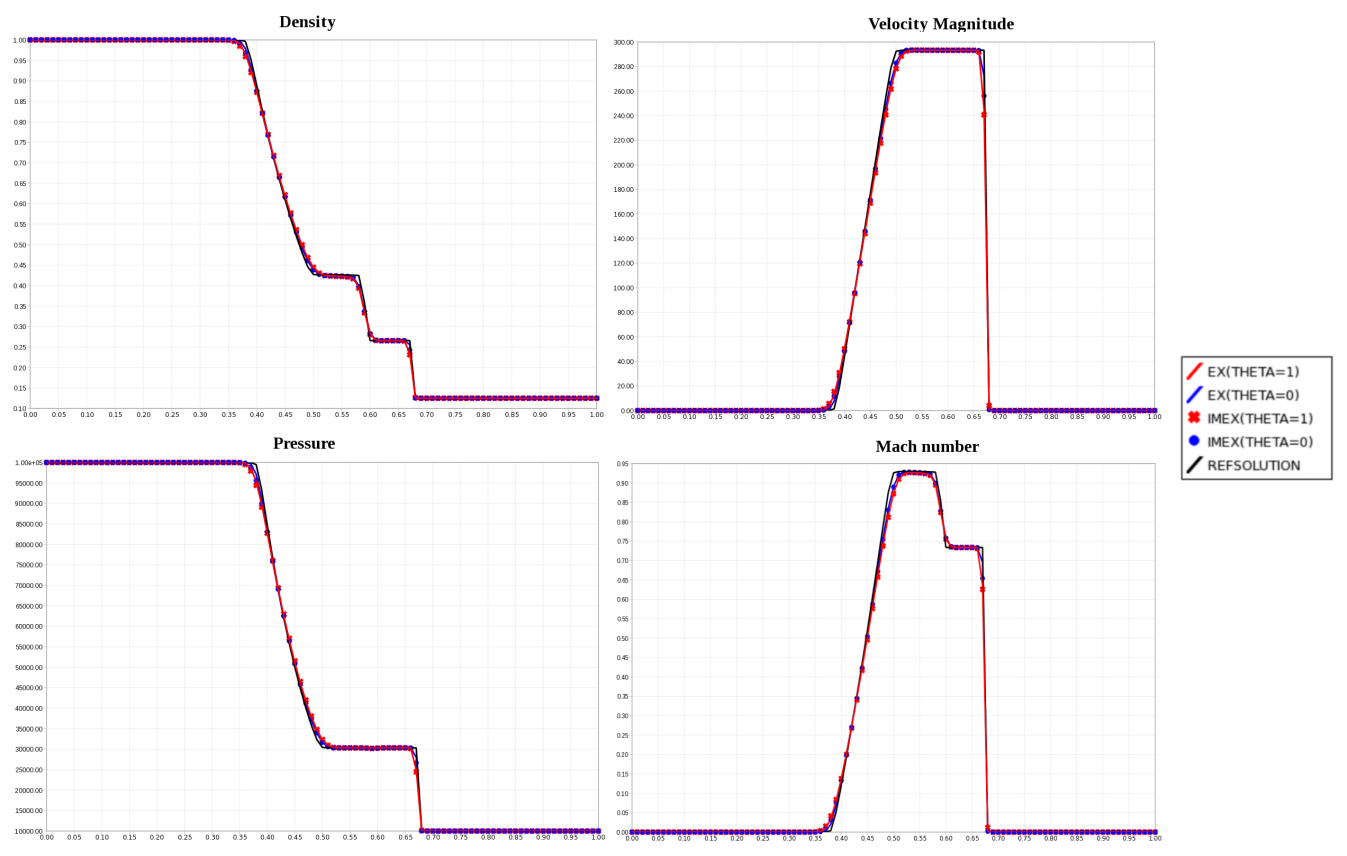

Figure 7: 1D Sod shock tube test case. Profile at $t=3.1 \times 10^{-4} \mathrm{~s}$ of the density (top left), velocity magnitude (top right), pressure (bottom left) and Mach number (bottom right) for the $\operatorname{EX}(\theta=1), \operatorname{EX}(\theta=0), \operatorname{IMEX}(\theta=1)$, $\operatorname{IMEX}(\theta=0)$ using a 1000-cell grid, together with reference solution.

Table 3: 2D Riemann problem test case. Comparison of the number of time steps and CPU time necessary for reaching $t=0.4 \mathrm{~s}$ with a $50 \times 50$-cell Cartesian grid with $\operatorname{EX}(\theta=1), \operatorname{EX}(\theta=0), \operatorname{IMEX}(\theta=1)$ and $\operatorname{IMEX}(\theta=0)$.

\begin{tabular}{ccccc}
\hline Numerical scheme & $\operatorname{EX}(\theta=1)$ & $\operatorname{EX}(\theta=0)$ & $\operatorname{IMEX}(\theta=1)$ & $\operatorname{IMEX}(\theta=0)$ \\
\hline Number of iterations & 323 & 343 & 216 & 218 \\
CPU time (s) & 2.59 & 2.79 & 10.28 & 10.33 \\
\hline
\end{tabular}

center of the domain shape is better captured with coarse meshes when one uses the corrected schemes $(\theta=0)$. A 1D cut along the axis $y=x$ as depicted in figure 10, also corroborates this observation: the approximation obtained with $\operatorname{EX}(\theta=0)$ and $\operatorname{IMEX}(\theta=0)$ schemes are closer to the $200 \times 200$-cell reference solution thanks to the numerical diffusion reduction. Nonetheless, we observe on a 1D cut along the $x=0.75$ axis in figure 11 a spurious overshot for both density and pressure located at the shock front with $\operatorname{EX}(\theta=0)$ and $\operatorname{IMEX}(\theta=0)$. This suggests that a small value of $\theta$ allows to improve the precision of the scheme by reducing the numerical diffusion but it may cause overshoots if the value of $\theta$ becomes too small relatively to the local behavior of flow. In all our numerical experiments the scheme seems to remain stable for any value of $\theta \in(0,1)$. Let us note that even if the pressure gradient is given a centred treatment $(\theta=0)$, the transport step introduce some numerical diffusion (independent of $M$ ) that stabilize the scheme see $(32)$.

In table 3 we observe that the choice of $\theta$ does not impact the number of time steps and CPU time. For this case, while the number of time steps is slightly reduced by about $30 \%$, the semi-implicit schemes are much slower due to the time required for solving the linear system involved with the schemes.

As a partial conclusion of this section, we can observe that for tests that strongly involve the compressibility of the fluid both semi-implicit and explicit schemes seem to be very robust, independantly of the choice of $\theta$ within $[0,1]$. However, if the low Mach correction is too important, i.e. the value of $\theta$ is too close to 0 we witnessed a deterioration of the numerical approximation with the appearance of overshoots in the vicinity of shock fronts. Then some numerical criterion may be constructed with good properties, $\theta_{i j}=\min \left(\frac{\left|u_{i j}^{*}\right|}{\max \left(c_{i}^{n}, c_{j}^{n}\right)}, 1\right)$ for instance. We also observed that the benefit in terms of CPU time of the semi-implicit scheme vanishes when 


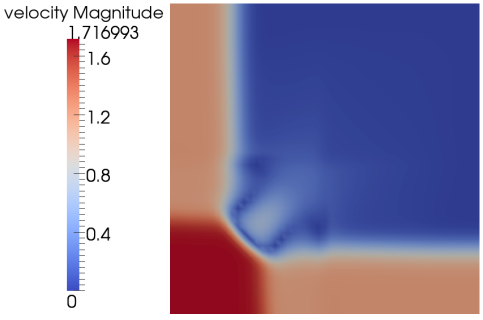

(a)

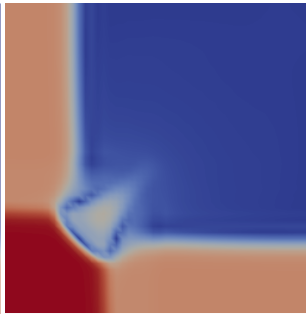

(b)

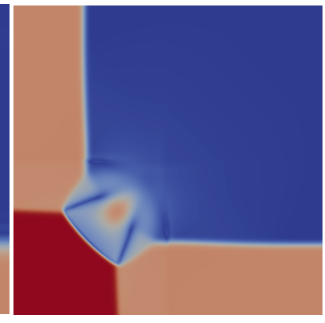

(c)
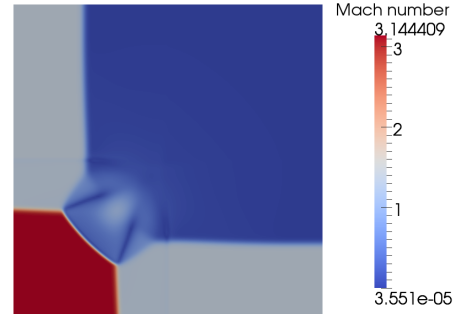

(d)

Figure 8: 2D Riemann problem with a Cartesian mesh. Profile at $t=0.4 \mathrm{~s}$ of the velocity magnitude for (a) $\operatorname{EX}(\theta=1)$, (b) $\operatorname{EX}(\theta=0)$ with a $50 \times 50$-cell mesh, (c) velocity magnitude and (d) Mach number with $\operatorname{EX}(\theta=1)$ using a $200 \times 200$ mesh.
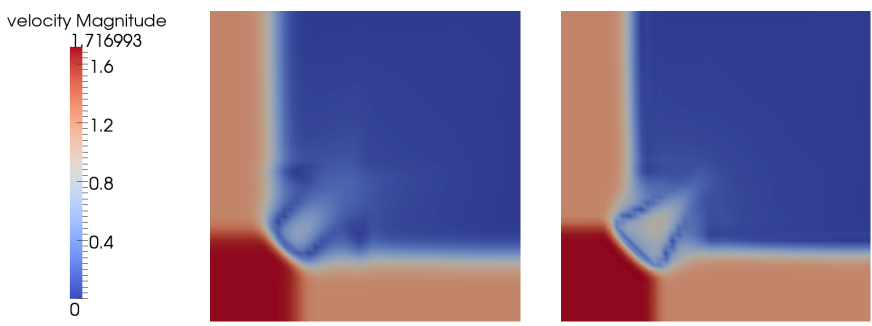

Figure 9: 2D Riemann problem test case. Profile at $t=0.4 \mathrm{~s}$ of the velocity magnitude for $\operatorname{IMEX}(\theta=1)$ (left) and $\operatorname{IMEX}(\theta=0)$ (right) on a $50 \times 50$-cell Cartesian mesh.

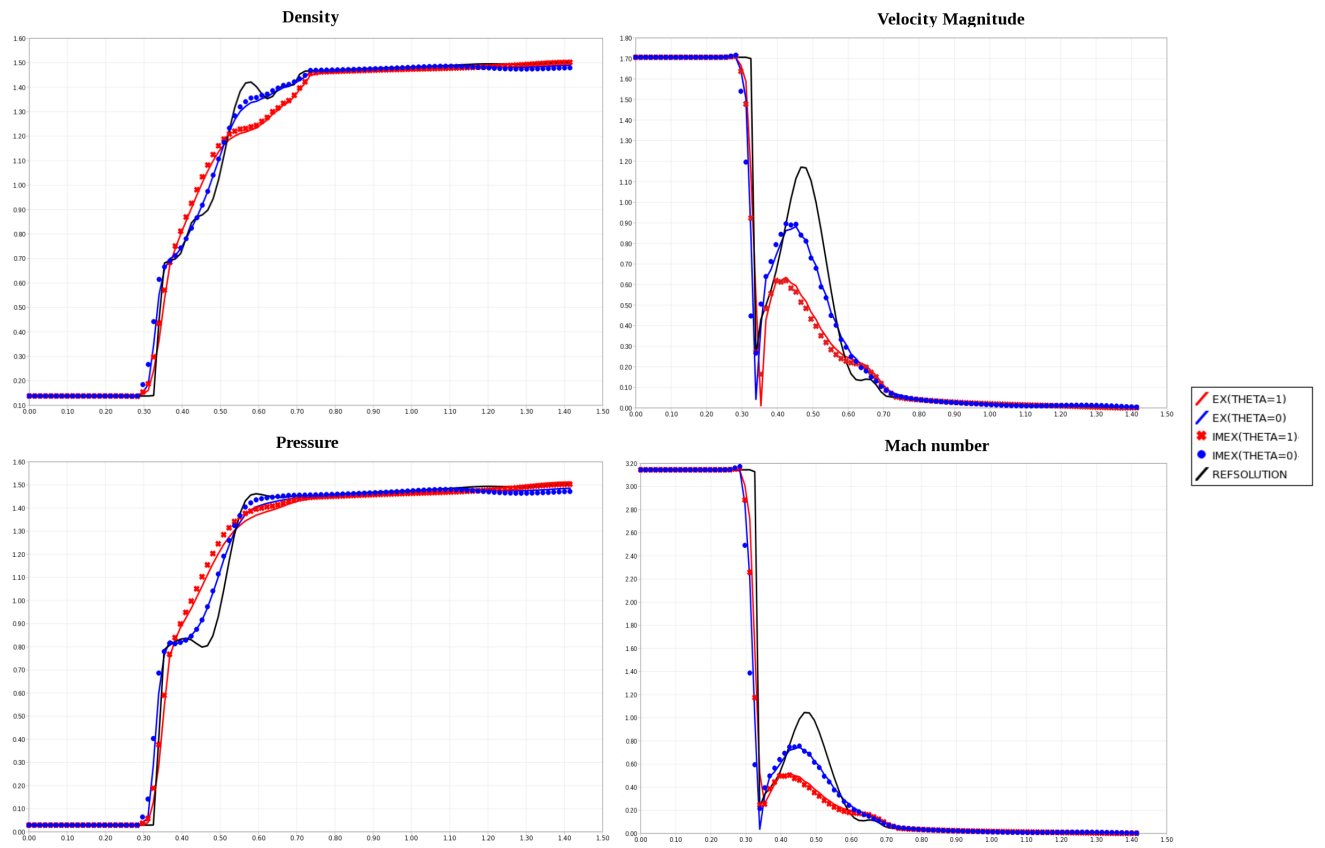

Figure 10: 2D Riemann problem test case. Cut profile along $y=x$ at $t=0.4 \mathrm{~s}$ of the density, velocity magnitude, pressure and Mach number for $\operatorname{EX}(\theta=1), \operatorname{EX}(\theta=0), \operatorname{IMEX}(\theta=1)$ and $\operatorname{IMEX}(\theta=0)$ using a $50 \times 50$ mesh together with the $200 \times 200$-cell reference solution. 


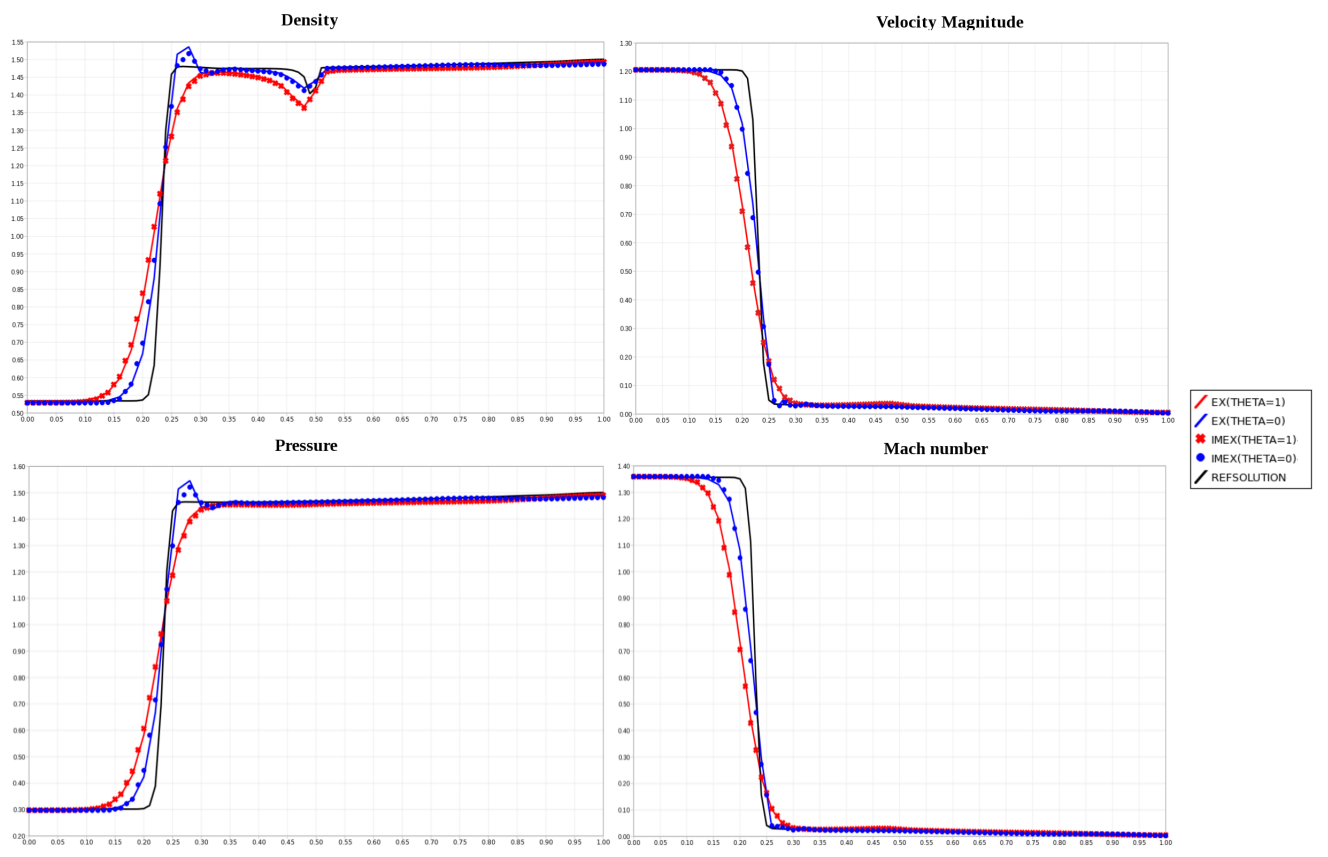

Figure 11: 2D Riemann problem test case. Cut profile along $x=0.75$ at $t=0.4 \mathrm{~s}$ of the density, velocity magnitude, pressure and Mach number for $\operatorname{EX}(\theta=1), \operatorname{EX}(\theta=0), \operatorname{IMEX}(\theta=1)$ and $\operatorname{IMEX}(\theta=0)$ using a $50 \times 50$ mesh together with the $200 \times 200$-cell reference solution.

the Mach number becomes of order 1.

The implementation of the criterion on $\theta$ to recover a discrete entropy inequality does not allow to recover a good low Mach behaviour as it was expected from its low Mach analysis. Finding from a theoretical point of view a criterion on $\theta$ that allows to recover a good low Mach behaviour and avoid spurious phenomenon that may occur if $\theta$ is too small for a given configuration is still an open problem.

Remark 6. The robustness of the scheme with respect to the modification $\theta \geq 0$ seems to be linked to the Lagrange-Projection decomposition approach. Indeed, numerical evidences not presented here show that a modified relaxation scheme written in Eulerian coordinates is unstable outside of the low-Mach regime for value of $\theta$ that are too small. For instance, The Sod shock test tube require $\theta>0.63$ to be stable.

\section{Conclusion}

We proposed a conservative operator splitting based Lagrange-Projection like numerical strategy for approximating the gas dynamics that decouples acoustic and transport phenomenons. The operator splitting scheme is positive for the density, the internal energy and entropic under classical CFL conditions. For one-dimensional problem, this procedure is equivalent to a Lagrange-Projection discretization. We presented an analysis of the way the truncation error depends on the Mach number for one-dimensional problems. In the low Mach regime, the truncation error of the scheme showed to be non-uniform with respect to the Mach number $M$. This allowed us to modify the operator splitting scheme in order to recover a uniform truncation error in term of $M$ by altering the numerical flux in the acoustic approximation. We showed that this modification can be obtained thanks to a simple approximate Riemann solver that is consistent with the integral form of the PDEs. This modified operator splitting scheme is conservative and endowed with good stability properties with respect to the positivity of the density, the internal energy under classical acoustic CFL conditions that depend on $M$. The resulting scheme allows to deal with tests where the flow regime may vary from low to high Mach values.

We showed that this splitting strategy has a natural extension to multi-dimensional problems discretized over unstructured meshes. A simple and efficient semi-implicit scheme that is stable under CFL conditions based on the material velocity is also proposed and leads to an all-regime numerical scheme, following the ideas 
paved by [6] for one-dimensional problems.

Future developments include extensions to high-order methods and approximation of other systems for the simulation of multi-material flows.

\section{Acknowledgement}

The authors would like to thank Pierre-Arnaud Raviart for sharing his personal notes and for the many rich discussions that brought an invaluable contribution to this work. 


\section{A Classical Lagrange-Projection for one-dimensional gas dynamics}

In this section we briefly recall the classical Lagrange-Projection (or Lagrange-Remap) procedure for deriving a Finite Volume discretization within a one-dimensional framework. For a detailed description we refer the reader to [15, 13. Let $(X, t) \mathbb{R} \times\left[t^{n}, t^{n}+\Delta t\right) \mapsto \chi$ be the mapping defined by

$$
\partial_{t} \chi=u(\chi(X, t), t), \quad \chi\left(X, t=t^{n}\right)=X .
$$

The pair $(X, t)$ is usually referred to as the Lagrangian system of coordinates: a particle of fluid at the position $X$ at instant $t=t^{n}$ will be located at $x=\chi(X, t), t \in\left[t^{n}, t^{n}+\Delta t\right]$. If $(x, t) \mapsto b$ is a mapping that provides an Eulerian representation of a parameter $b$, one defines a Lagrangian representation of $b$ as the function $(X, t) \mapsto b^{\mathrm{Lag}}$ by setting $b^{\mathrm{Lag}}(X, t)=b(\chi(X, t), t)$. The system (3) is equivalent to

$$
\partial_{t} \mathbf{V}^{\mathrm{Lag}}(X, t)+\tau^{\mathrm{Lag}}\left(X, t^{n}\right) \partial_{X} \mathbf{F}^{\mathrm{Lag}}\left(\mathbf{V}^{\mathrm{Lag}}\right)(X, t)=0, \quad\left\{\begin{array}{l}
\mathbf{V}^{\mathrm{Lag}}=\left(\tau^{\mathrm{Lag}}, u^{\mathrm{Lag}}, v^{\mathrm{Lag}}, E^{\mathrm{Lag}}\right)^{T}, \\
\mathbf{F}^{\mathrm{Lag}}\left(\mathbf{V}^{\mathrm{Lag}}\right)=\left(-u^{\mathrm{Lag}}, p^{\mathrm{Lag}}, 0, p^{\mathrm{Lag}} u^{\mathrm{Lag}}\right)^{T} .
\end{array}\right.
$$

It is common to introduce a mass coordinate $m$ defined by $\mathrm{d} m=\rho\left(X, t^{n}\right) \mathrm{d} X$ in order to obtain the equivalent conservation laws (with a slight abuse of notation)

$$
\partial_{t} \mathbf{V}^{\mathrm{Lag}}(m, t)+\partial_{m} \mathbf{F}^{\mathrm{Lag}}\left(\mathbf{V}^{\mathrm{Lag}}\right)(m, t)=0 .
$$

Straightforward calculations show that (68) (which is nothing but (6) ) is hyperbolic over the phase space $\Omega^{\mathrm{Lag}}=\left\{\left(\tau^{\mathrm{Lag}}, u^{\mathrm{Lag}}, v^{\mathrm{Lag}}, E^{\mathrm{Lag}}\right)^{T} \in \mathbb{R}^{4}, \tau^{\mathrm{Lag}}>0, e^{\mathrm{Lag}}>0\right\}$, with eigenvalues given by $\lambda_{1}^{\mathrm{Lag}}=-\rho c<\lambda_{2}^{\mathrm{Lag}}=$ $0<\lambda_{3}^{\mathrm{Lag}}=\rho c$, where $c$ still denotes the Eulerian sound speed. Here again, the extreme characteristic fields associated with $\lambda_{1}^{\mathrm{Lag}}$ and $\lambda_{3}^{\mathrm{Lag}}$ are genuinely non linear while the intermediate characteristic field associated with $\lambda_{2}^{\mathrm{Lag}}$ is linearly degenerate. It is important to note that the material transport phenomenons are frozen in system (68) which explains why the characteristics speeds of the system only involve the sound velocity $c$.

Before going any further, we introduce classical notations: let $\Delta t>0$ and $\Delta x>0$ be respectively the time and space steps. We define the Eulerian mesh interfaces $x_{j+1 / 2}=j \Delta x$ for $j \in \mathbb{Z}$, and the intermediate times $t^{n}=n \Delta t$ for $n \in \mathbb{N}$. If $b$ is a fluid parameter, in the sequel, we will note $b_{j}^{n}$ (resp. $b_{j}^{n+1}$ ) the approximate value $b$ respectively within the $j^{\text {th }}$ Eulerian cell $\left[x_{j-1 / 2}, x_{j+1 / 2}\right)$ at instant $t=t^{n}\left(\right.$ resp. $\left.t=t^{n+1}\right)$. We need to introduce a moving Lagrangian mesh (with respect to the Eulerian mesh) whose cell $j$ at instant $t^{n}$ is $\left[x_{j-1 / 2}, x_{j+1 / 2}\right.$ ) and at instant $t=t^{n+1}$ is $\left[x_{j-1 / 2}^{*}, x_{j+1 / 2}^{*}\right)$. The value of the parameter $b$ at instant $t^{n}$ (resp. $\left.t=t^{n+1}\right)$ in the Lagrangian cell $j$ is noted $b_{j}^{\text {Lag }}$ (resp. $b_{j}^{n+1-}$ ). Given a fluid state $(\rho, \rho u, \rho v, \rho E)_{j}^{n}, j \in \mathbb{Z}$ at instant $t^{n}$, the Lagrange-Projection strategy proposes the following update procedure.

1. Build the discrete Lagrangian fluid state at instant $t^{n}$ by setting $\left(\mathbf{V}^{\mathrm{Lag}}\right)_{j}=\left(\tau_{j}^{n}, u_{j}^{n}, v_{j}^{n}, E_{j}^{n}\right)$;

2. Update the Lagrangian fluid state into the value $\left(\mathbf{V}^{\mathrm{Lag}}\right)_{j}^{n+1-}=\left(\tau_{j}^{n+1-}, u_{j}^{n+1-}, v_{j}^{n+1-}, E_{j}^{n+1-}\right)$ by approximating the solution of (68);

3. Build the updated value $(\rho, \rho u, \rho v, \rho E)_{j}^{n+1}$ by remapping the Lagrangian state $\left(\mathbf{V}^{\mathrm{Lag}}\right)_{j}^{n+1-}$ onto the Eulerian mesh.

The Lagrangian step $\left(t^{n} \rightarrow t^{n+1-}\right)$

We propose to approximate the solution of $(68)$ using the acoustic scheme [12, 13]. This leads to

$$
\left\{\begin{array}{c}
\tau_{j}^{n+1-}=\tau_{j}^{n}+\frac{\Delta t}{\Delta x} \tau_{j}^{n}\left(u_{j+1 / 2}^{*}-u_{j-1 / 2}^{*}\right), \\
u_{j}^{n+1-}=u_{j}^{n}-\frac{\Delta t}{\Delta x} \tau_{j}^{n}\left(p_{j+1 / 2}^{*}-p_{j-1 / 2}^{*}\right), \\
v_{j}^{n+1-}=v_{j}^{n}, \\
E_{j}^{n+1-}=E_{j}^{n}-\frac{\Delta t}{\Delta x} \tau_{j}^{n}\left((p u)_{j+1 / 2}^{*}-(p u)_{j-1 / 2}^{*}\right),
\end{array}\right.
$$

where the interfaces terms are defined by

$$
\begin{array}{rlrl}
u_{j+1 / 2}^{*} & =\frac{\left(u_{j}^{n}+u_{j+1}^{n}\right)}{2}+\frac{1}{2 a_{j+1 / 2}^{n}}\left(p_{j}^{n}-p_{j+1}^{n}\right), & & p_{j+1 / 2}^{*}=\frac{\left(p_{j}^{n}+p_{j+1}^{n}\right)}{2}+\frac{a_{j+1 / 2}^{n}}{2}\left(u_{j}^{n}-u_{j+1}^{n}\right), \\
(p u)_{j+1 / 2}^{*} & =p_{j+1 / 2}^{*} u_{j+1 / 2}^{*}, & a_{j+1 / 2}^{n}=\max \left((\rho c)_{j}^{n},(\rho c)_{j+1}^{n}\right) .
\end{array}
$$


The acoustic scheme (69) with (70) provides the same update of the flow variable as the scheme (9) with (10). Let us mention that a direct proof of stability for the acoustic scheme is available in [12] under the CFL criterion (17).

The projection (or remapping) step $\left(t^{n+1-} \rightarrow t^{n+1}\right)$

The aim of this step is to project the solution obtained at the end of the Lagrangian step onto the Eulerian cells $\left[x_{j-1 / 2}, x_{j+1 / 2}\right)$. If one notes $\mathbb{1}_{\left[x_{j-1 / 2}^{*}, x_{j+1 / 2}^{*}\right)}$ the characteristic function of $\left[x_{j-1 / 2}^{*}, x_{j+1 / 2}^{*}\right)$, a standard way to achieve to goal consists in: first, approximating the position of the Lagrangian mesh interfaces at instant $t^{n+1}$ by setting $x_{j+1 / 2}^{*}=x_{j+1 / 2}+u_{j+1 / 2}^{*} \Delta t$; second reaveraging the conservative variable unknowns over the Eulerian mesh by setting [15]

$$
\varphi_{j}^{n+1}=\frac{1}{\Delta x} \int_{x_{j-1 / 2}}^{x_{j+1 / 2}}\left[\sum_{j \in \mathbb{Z}} \varphi_{j}^{n+1-} \mathbb{1}_{\left[x_{j-1 / 2}^{*}, x_{j+1 / 2}^{*}\right)}(x)\right] \mathrm{d} x, \quad \text { where } \varphi \in\{\rho, \rho u, \rho v, \rho E\} .
$$

Noting $\Delta x_{j}^{*}=x_{j+1 / 2}^{*}-x_{j-1 / 2}^{*}$ and $\varepsilon(j, n)=-\operatorname{sign}\left(u_{j+1 / 2}^{*}\right) 1 / 2$ one obtains the update formula

$$
\begin{aligned}
\varphi_{j}^{n+1} & =\frac{1}{\Delta x}\left\{\Delta x_{j}^{*} \varphi_{j}^{n+1-}-\Delta t\left(u_{j+1 / 2}^{*} \varphi_{j+1 / 2+\varepsilon(j, n)}^{n+1-}-u_{j-1 / 2}^{*} \varphi_{j-1 / 2+\varepsilon(j-1, n)}^{n+1-}\right)\right\} \\
& =\frac{\Delta t}{\Delta x}\left(u_{j+1 / 2}^{*}-u_{j-1 / 2}^{*}\right) \varphi_{j}^{n+1-}-\frac{\Delta t}{\Delta x}\left(u_{j+1 / 2}^{*} \varphi_{j+1 / 2+\varepsilon(j, n)}^{n+1-}-u_{j-1 / 2}^{*} \varphi_{j-1 / 2+\varepsilon(j-1, n)}^{n+1-}\right)
\end{aligned} .
$$

The update formula 72 matches the classic upwind scheme. Consequently this is the same numerical scheme as (14).

\section{B Approximate Riemann solvers: Harten Lax and van Leer formal- ism}

We briefly recall the Harten, Lax and van Leer formalism associated with the numerical approximation of the solutions $(x, t) \in \mathbb{R} \times[0,+\infty) \mapsto \mathbf{U} \in \mathbb{R}^{m}$ of the general hyperbolic system of conservation laws

$$
\partial_{t} \mathbf{U}+\partial_{x} \mathbf{G}(\mathbf{U})=0, \quad x \in \mathbb{R}, t>0,
$$

by means of the so-called approximate Riemann solvers and Godunov-type methods, where $\mathbf{G}: \mathbb{R}^{m} \rightarrow \mathbb{R}^{m}$ is a smooth function. System 73 is supplemented with the validity of an entropy inequality

$$
\partial_{t} \eta(\mathbf{U})+\partial_{x} q(\mathbf{U}) \leq 0
$$

where $\mathbf{U} \mapsto(\eta, q)$ is a strictly convex entropy-entropy flux pair (see [15]).

Solving the Riemann problem amounts to find the solution of $(73)$ with the following piecewise constant initial data

$$
\mathbf{U}(x, t=0)=\left\{\begin{array}{lll}
\mathbf{U}_{L}, & \text { if } & x<0, \\
\mathbf{U}_{R}, & \text { if } & x>0,
\end{array}\right.
$$

for any given $\mathbf{U}_{L}$ and $\mathbf{U}_{R}$ in the phase space. It is well-known that the exact Riemann solution $\mathbf{U}\left(x / t ; \mathbf{U}_{L}, \mathbf{U}_{R}\right)$ is self-similar, i.e. depends only on the ration $x / t$. In order to approximate this solution, we consider a (selfsimilar) simple approximate Riemann solver $\mathbf{U}_{\mathrm{RP}}\left(\frac{x}{t} ; \mathbf{U}_{L}, \mathbf{U}_{R}\right)$ made of $l+1$ intermediate states $\mathbf{U}_{k}$ separated by discontinuities propagating with velocities $\lambda_{1} \leq \cdots \leq \lambda_{l}$, namely

$$
\mathbf{U}_{\mathrm{RP}}\left(\frac{x}{t} ; \mathbf{U}_{L}, \mathbf{U}_{R}\right)=\left\{\begin{array}{lll}
\mathbf{U}_{1}=\mathbf{U}_{L}, & \text { if } \quad x / t<\lambda_{1}, \\
\vdots & \text { if } \quad \lambda_{k-1}<x / t<\lambda_{k}, \\
\mathbf{U}_{k}, & & \\
\vdots & \text { if } \quad x / t>\lambda_{l} .
\end{array}\right.
$$


From [17, 1], if $\Delta x=\frac{1}{2}\left(\Delta x_{L}+\Delta x_{R}\right)$ with $\Delta x_{L}>0, \Delta x_{R}>0$ and $\Delta t>0$ are respectively space and time steps that verify the CFL condition

$$
\max _{1 \leq k \leq l}\left|\lambda_{k}\right| \frac{\Delta t}{\min \left(\Delta x_{L}, \Delta x_{R}\right)} \leq \frac{1}{2}
$$

such an approximate Riemann solver is said to be consistent with the integral form of 73 over the interval $\left[\frac{-\Delta x_{L}}{2}, \frac{\Delta x_{R}}{2}\right] \times[0, \Delta t]$ if $\iint_{\left[\frac{-\Delta x_{L}}{2}, \frac{\Delta x_{R}}{2}\right] \times[0, \Delta t]}\left[\partial_{t} \mathbf{U}_{\mathrm{RP}}+\partial_{x} \mathbf{G}\left(\mathbf{U}_{\mathrm{RP}}\right)\right] \mathrm{d} x \mathrm{~d} t=0$, in other words if

$$
\mathbf{G}\left(\mathbf{U}_{R}\right)-\mathbf{G}\left(\mathbf{U}_{L}\right)=\sum_{k=1}^{l} \lambda_{k}\left(\mathbf{U}_{k+1}-\mathbf{U}_{k}\right) .
$$

Regarding the consistency with the entropy inequality (74, , the simple approximate Riemann solver is said to be consistent with the integral form of 74 if and only if under the CFL condition 76 we have

$$
q\left(\mathbf{U}_{R}\right)-q\left(\mathbf{U}_{L}\right) \leq \sum_{k=1}^{l} \lambda_{k}\left(\eta\left(\mathbf{U}_{k+1}\right)-\eta\left(\mathbf{U}_{k}\right)\right) .
$$

Hereafter and using classic notations, $\left(\Delta x_{j}\right)_{j \in \mathbb{Z}}$ and $\Delta t$ represent the space steps and constant time step of the mesh under consideration to define the approximate solutions. More precisely, we define the mesh interfaces $x_{j+1 / 2}=x_{j-1 / 2}+\Delta x_{j}$ for $j \in \mathbb{Z}$, the intermediate times $t^{n}=n \Delta t$ for $n \in \mathbb{N}$, and we note $\mathbf{U}_{j}^{n}$ the approximate value of $\mathbf{U}$ at time $t^{n}$ and on the cell $\left[x_{j-1 / 2}, x_{j+1 / 2}\right)$. For $n=0$ and $j \in \mathbb{Z}$, we set $\mathbf{U}_{j}^{0}=\frac{1}{\Delta x} \int_{x_{j-1 / 2}}^{x_{j+1 / 2}} \mathbf{U}_{0}(x) \mathrm{d} x$ where $\mathbf{U}_{0}(x)$ is the initial condition. Then, the explicit in time Godunov-type scheme reads

$$
\left\{\begin{array}{l}
\mathbf{U}_{j}^{n+1}=\mathbf{U}_{j}^{n}-\frac{\Delta t}{\Delta x_{j}}\left(\mathbf{G}_{j+\frac{1}{2}}^{n}-\mathbf{G}_{j-\frac{1}{2}}^{n}\right) \\
\mathbf{G}_{j+\frac{1}{2}}^{n}=\mathbf{G}\left(\mathbf{U}_{j}^{n}, \mathbf{U}_{j+1}^{n}\right)
\end{array}\right.
$$

with

$$
\mathbf{G}\left(\mathbf{U}_{L}, \mathbf{U}_{R}\right)=\frac{1}{2}\left[\mathbf{G}\left(\mathbf{U}_{L}\right)+\mathbf{G}\left(\mathbf{U}_{R}\right)-\sum_{k=1}^{l}\left|\lambda_{k}\right|\left(\mathbf{U}_{k+1}-\mathbf{U}_{k}\right)\right] .
$$

Moreover, if the simple approximate Riemann solver is consistent with the entropy inequality $(74)$, then the numerical scheme defined by (79) satisfies the following discrete entropy inequality

$$
\left\{\begin{array}{r}
\eta\left(\mathbf{U}_{j}^{n+1}\right) \leq \eta\left(\mathbf{U}_{j}^{n}\right)-\frac{\Delta t}{\Delta x_{j}}\left(q_{j+\frac{1}{2}}^{n}-q_{j-\frac{1}{2}}^{n}\right), \\
q_{j+\frac{1}{2}}^{n}=\widetilde{q}\left(\mathbf{U}_{j}^{n}, \mathbf{U}_{j+1}^{n}\right),
\end{array}\right.
$$

with

$$
\widetilde{q}\left(\mathbf{U}_{L}, \mathbf{U}_{R}\right)=\frac{1}{2}\left[q\left(\mathbf{U}_{L}\right)+q\left(\mathbf{U}_{R}\right)-\sum_{k=1}^{l}\left|\lambda_{k}\right|\left(S\left(\mathbf{U}_{k+1}\right)-S\left(\mathbf{U}_{k}\right)\right)\right] .
$$

The CFL condition associated with this (explicit in time) Godunov-type scheme reads

$$
\max _{1 \leq k \leq l}\left|\lambda_{k}\left(\mathbf{U}_{j}^{n}, \mathbf{U}_{j+1}^{n}\right)\right| \frac{\Delta t}{\min \left(\Delta x_{j}, \Delta x_{j+1}\right)} \leq \frac{1}{2},
$$

for all $j$. Again, we refer to 17, 1 for more details. To conclude this paragraph, let us observe that the numerical flux $\mathbf{G}\left(\mathbf{U}_{L}, \mathbf{U}_{R}\right)$ and the entropy numerical flux $\widetilde{q}\left(\mathbf{U}_{L}, \mathbf{U}_{R}\right)$ are clearly consistent in the classical sense, namely $\mathbf{G}(\mathbf{U}, \mathbf{U})=\mathbf{G}(\mathbf{U})$ and $\widetilde{q}(\mathbf{U}, \mathbf{U})=q(\mathbf{U})$ provided that the intermediate states of the approximate Riemann solver are such that $\mathbf{U}_{k}=\mathbf{U}$ for all $k=1, \ldots, l$ as soon as $\mathbf{U}_{L}=\mathbf{U}_{R}=\mathbf{U}$. 


\section{Riemann problem for the relaxation approximation of the acous- tic system}

We consider the Suliciu relaxation approximation of the Lagrangian gas dynamics equations expressed using a mass coordinate. The system reads

$$
\left\{\begin{aligned}
\partial_{t} \tau-\partial_{m} u & =0, \\
\partial_{t} u+\partial_{m} \Pi & =0, \\
\partial_{t} v & =0, \\
\partial_{t} E+\partial_{m}(\Pi u) & =0, \\
\partial_{t} \Pi+a^{2} \partial_{m} u & =\lambda(p-\Pi),
\end{aligned}\right.
$$

where $a$ is a constant that verifies the subcharacteristic condition $a>\max (\rho c)$ in order to prevent instabilities (see for instance [3] for a rigorous proof). It is easy to prove that the convective part of 83 is strictly hyperbolic with three eigenvalues given by $-a, 0$ and $a$ which correspond to linearizations of the exact eigenvalues $-\rho c$, 0 and $\rho c$ for system (67). Interestingly, the characteristic fields are linearly degenerate, which allows to solve analytically the Riemann problem associated with 83 with $\lambda=0$. More precisely, the exact Riemann solution

$$
\overline{\mathbf{W}}\left(\frac{m}{t} ; \overline{\mathbf{U}}_{L}, \overline{\mathbf{U}}_{R}\right)=(\tau, u, v, E, \Pi)^{T}\left(\frac{m}{t} ; \overline{\mathbf{U}}_{L}, \overline{\mathbf{U}}_{R}\right)
$$

associated with given left state $\overline{\mathbf{U}}_{L}=(\tau, u, v, E, \Pi)_{L}^{T}$ and right state $\overline{\mathbf{U}}_{R}=(\tau, u, v, E, \Pi)_{R}^{T}$, is made of three contact discontinuities propagating with velocities $-a, a$ and 0 and separating two intermediate states $\overline{\mathbf{U}}_{L}^{*}$ and $\overline{\mathbf{U}}_{R}^{*}$, namely

$$
\overline{\mathbf{W}}\left(\frac{m}{t} ; \overline{\mathbf{U}}_{L}, \overline{\mathbf{U}}_{R}\right)= \begin{cases}\overline{\mathbf{U}}_{L}, & \text { if } \frac{m}{t}<-a, \\ \overline{\mathbf{U}}_{L}^{*}, & \text { if }-a<\frac{m}{t}<0, \\ \overline{\mathbf{U}}_{R}^{*}, & \text { if } 0<\frac{m}{t}<a, \\ \overline{\mathbf{U}}_{R}, & \text { if } \frac{m}{t}>a .\end{cases}
$$

The intermediate states are easily recovered from the following formulas

$$
\begin{cases}u^{*}=u_{L}^{*}=u_{R}^{*}=\frac{u_{R}+u_{L}}{2}-\frac{\Pi_{R}-\Pi_{L}}{2 a}, & \Pi_{L}^{*}=\Pi_{R}^{*}=\Pi^{*}=\frac{\Pi_{R}+\Pi_{L}}{2}-a \frac{u_{R}-u_{L}}{2}, \\ v_{L}^{*}=v_{L}, & v_{R}^{*}=v_{R}, \\ \tau_{L}^{*}=\tau_{L}+\frac{u^{*}-u_{L}}{a}, \frac{u_{R}-u^{*}}{a}, \\ E_{L}^{*}=E_{L}+\frac{1}{a}\left(p_{L} u_{L}-u^{*} \Pi^{*}\right), & E_{R}^{*}=E_{R}-\frac{1}{a}\left(p_{R} u_{R}-u^{*} \Pi^{*}\right) .\end{cases}
$$

Then, setting $\mathbf{U}_{L}=(\tau, u, v, E)^{T}$, the classical scheme can be understood in the Harten, Lax and van Leer formalism by considering the following approximate Riemann solver $\mathbf{W}\left(\frac{m}{t} ; \mathbf{U}_{L}, \mathbf{U}_{R}\right)$ obtained by simply extracting the first four components from $\overline{\mathbf{W}}\left(\frac{m}{t} ; \overline{\mathbf{U}}_{L}, \overline{\mathbf{U}}_{R}\right)$, in which we take $\Pi$ at equilibrium, namely

$$
\Pi_{L}=p_{L}, \quad \Pi_{R}=p_{R} .
$$

More precisely, we have

$$
\mathbf{W}\left(\frac{m}{t} ; \mathbf{U}_{L}, \mathbf{U}_{R}\right)= \begin{cases}\mathbf{U}_{L}, & \frac{m}{t}<-a, \\ \mathbf{U}_{L}^{*}, & -a<\frac{m}{t}<0, \\ \mathbf{U}_{R}^{*}, & 0<\frac{m}{t}<a, \\ \mathbf{U}_{R}, & \frac{m}{t}>a,\end{cases}
$$

where the intermediate states are given by (85), together with $\Pi_{L}=p_{L}$ and $\Pi_{R}=p_{R}$.

\section{References}

[1] F. Bouchut. Nonlinear stability of finite volume methods for hyperbolic conservation laws, and well-balanced schemes for sources. Frontiers in Mathematics series. Birkhäuser, 2004. 
[2] C. Chalons and F. Coquel. Navier-stokes equations with several independant pressure laws and explicit predictor-corrector schemes. Numerisch Math, 101(3):pp. 451-478, 2005.

[3] C. Chalons and J.-F. Coulombel. Relaxation approximation of the Euler equations. J. Math. Anal. Appl., 348(2):pp. 872-893, 2008.

[4] C. Chalons, M. Girardin, and S. Kokh. Large time step and asymptotic preserving numerical schemes for the gas dynamics equations with source terms. SIAM J. Sci. Comput., 35(6):pp. a2874-a2902, 2013.

[5] Ph. Colella and K. Pao. A projection method for low speed flows. J. Comp. Phys., 149(2):pp. 245-269, 1999.

[6] F. Coquel, Q. L. Nguyen, M. Postel, and Q. H. Tran. Entropy-satisfying relaxation method with large time-steps for Euler IBVPs. Math. Comput., 79(271):pp. 1493-1533, 2010.

[7] F. Cordier, P. Degond, and A. Kumbaro. An Asymptotic-Preserving all-speed scheme for the Euler and NavierStokes equations. J. Comp. Phys., 231(17):pp. 5685-5704, 2012.

[8] P. Degond and M. Tang. All speed method for the Euler equation in the low mach number limit. Communications in Computational Physics, 10:pp. 1-31, 2011.

[9] P. Degond, S. Jin, and J.-G. Liu. Mach-number uniform asymptotic-preserving gauge schemes for compressible flows. Bull. Inst. Math., Acad. Sin. (N.S.), 2(4):pp. 851-892, 2007.

[10] S. Dellacherie, P. Omnes and P.A. Raviart. Construction of modified Godunov type schemes accurate at any Mach number for the compressible Euler system. submitted, 2013.

[11] S. Dellacherie. Analysis of Godunov type schemes applied to the compressible euler system at low Mach number. J. Comp. Phys., 229(4):pp. 978-1016, 2010.

[12] B. Després. Inégalité entropique pour un solveur conservatif du système de la dynamique des gaz en coordonnées de lagrange. C. R. Acad. Sci. Paris, Série I, 324:pp. 1301-1306, 1997.

[13] B. Després. Lois de conservations Eulériennes, Lagrangiennes et méthodes numériques, volume 68 of Mathématiques et applications, SMAI. Springer, 2010.

[14] F. Dauvergne, J.-M. Ghidaglia, F. Pascal, and J.-M. Rovarch. Renormalization of the numerical diffusion for an upwind finite volume method. application to the simulation of Kelvin-Helmholtz instability. Finite volumes for complex applications. V. Proceedings of the 5th International Symposium, Aussois, June 2008, R. Eymard and J.-M. Hérard editors, pages pp. 321-328, 2008.

[15] E. Godlewski and P.-A. Raviart. Numerical approximation of hyperbolic systems of conservation laws. Springer, 1996.

[16] H. Guillard and C. Viozat. On the behavior of upwind schemes in the low Mach limit. Comp. EJ Fluid, 28:pp. 63-86, 1999.

[17] A. Harten, P.D. Lax, and B. Van Leer. On upstream differencing and godunov-type schemes for hyperbolic conservation laws. SIAM Review, 25:pp. 35-61, 1983.

[18] I. Toumi, A. Kumbaro, and H. Paillere. Approximate Riemann solvers and flux vector splitting schemes for two-phase flow. In VKI LS 1999-03, Computational Fluid Dynamics, 1999.

[19] J.-G. Liu J. Haack, S. Jin. An all-speed asymptotic-preserving method for the isentropic Euler and NavierStokes equations. Commun. Comp. Phys., 12:pp. 955-980, 2012.

[20] S. Jin. Runge-Kutta methods for hyperbolic conservation laws with stiff relaxation terms. J. Comp. Phys., 122(1):pp. 51-67, 1995.

[21] R. Klein. Semi-implicit extension of a Godunov-type scheme based on low Mach number asymptotics I: One-dimensional flow. J. Comp. Phys., 121(2):pp. 213-237, 1995. 
[22] P. D. Lax and X.-D. Liu. Solution of two-dimensional Riemann problems of gas dynamics by positive schemes. SIAM J. Sci. Comput., 19(2):pp. 319-340, 1998.

[23] M.-S. Liou. A sequel to AUSM, part ii: AUSM+-up for all speeds. J. Comp. Phys., 214(1):pp. 137-170, 2006.

[24] H. Paillère, C. Viozat, A. Kumbaro, and I. Toumi. Comparison of low mach number models for natural convection problems. Heat and Mass Transfer, 36(6):pp. 567-573, 2000.

[25] S. Schochet. Fast singular limits of hyperbolic PDEs. J. Differ. Equations, 114(2):pp. 476-512, 1994.

[26] G. A. Sod. Numerical methods in fluid dynamics. Initial and initial-boundary value problems. Cambridge: Cambridge University Press, 1985.

[27] I. Suliciu. On the thermodynamics of rate-type fluids and phase transitions. i. rate-type fluids. International Journal of Engineering Science, 36(9):pp. 921-947, 1998.

[28] B. Thornber, A. Mosedale, D. Drikakis, D. Youngs, and R.J.R. Williams. An improved reconstruction method for compressible flows with low Mach number features . J. Comp. Phys., 227(10):pp. 4873-4894, 2008 .

[29] E. Turkel. Preconditioned methods for solving the incompressible and low speed compressible equations. J. Comp. Phys., 72(2):pp. 277-298, 1987.

[30] H. Weyl. Shock waves in arbitrary fluids. Commun. Pure Appl. Math., 2:pp. 103-122, 1949. 